(Aus dem pharmakologischen Institut der Reichsuniversität Utrecht.)

\title{
Der Einfluss der Vagusreizung auf den Ablaur der Verdauungsbewegungen.
}

\author{
Röntgenversuche an der Rückenmarkskatze. \\ Von \\ Dr. Ph. Klee,
}

z. Z Assistenzarzt der medizinischen Klinik Tübingen.

(Mit 11 Textfiguren.)

Inhal tsübersicht.

Einleitung. . . . . . . . . . . . . . . . . 557

Methodik . . . . . . . . . . . . . . . . 558

1. Die Verdauungsbewegungen an der Rückenmarkskatze . . . . . . 562

a) Ohne Durchschneidung der oberen Nn. splanchnici . . . . . . . 562

b) Mit Durchschneidung der oberen Nn. splanchnici . . . . . . . . 569

2. Der Einfluss der Vagusreizung anf die Verdaungsbewegungen an der Rückenmarkskatze. . . . . . . . . . . . . . . . 572

a) Literatur . . . . . . . . . . . . . . . . 572

b) Versuchsanordnung. . . . . . . . . . . . . . . 574

c) Die Wirkung der Vagusreizung auf Magenperistaltik und Magenentleerung. . . . . . . . . . . . . . . . . . 576

d) Die Wirkung der Vagusreizung auf die Dünndarmbewegungen und die Weiterbeförderung des Dünndarminhaltes. . . . . . . . . 587

e) Versuche über das Verhalten des Dickdarms nach Vagusreizung . 591

3. Zusammenfassung der Ergebnisse ............. . 592

Die Bahnen, auf welchen den Magen-Darmbewegungen Impulse vom Zentralnervensystem übermittelt werden, sind die Fasern des Vagus, der Splanchnici und des Pelvicus. Über den allgemeinen Effekt dieser Nerven auf die Magen-Darmmuskulatur sind wir durch vielfache Untersuchungen unterrichtet. Wir wissen, dass Vagus und Pelvicus hauptsächlich einen erregenden, die Splanchnici einen hemmenden Einfluss ausüben. Welchen tatsächlichen Effekt diese gesteigerten oder gehemmten Bewegungen des Magens und Darmes auf die Weiter- 
beförderung ihres Inhaltes haben, dafür liegen uns nur wenige und meist auf einzelne Teile des Verdaungsrohres beschränkte Angaben vor. Dass wir aber nicht aus einer Verstärkung der Bewegungen der Magen-Darmmuskulatur ohne weiteres auf einen ebenso beschleunigten Transport der Ingesta schliessen können, ist bekannt. Wissen wir doch durch die Untersuchungen von Pawlow, Cohnheim, Bayliss und Starling, Cannon u. a., welch bedeutsame regulierende Rolle die lokalen Hemmungsreflexe chemischer oder mechanischer Art bei der Fortbewegung des Speisebreies spielen. Und dieser Punkt erscheint um so beachtenswerter, als durch eine abnorme Erregung oder Ausschaltung der genannten Nerven nicht nur Veränderungen der Verdauungsbewegungen, sondern auch der Sekretmenge der Verdauungsdrüsen entstehen, die dann ihrerseits eine Verstärkung oder Abschwächung der Chemoreflexe bedingen können. Die komplizierten Reflexe bei der Magenentleerung, speziell der Säurehemmungsreflex des Duodenums, erläutern diese Verhältnisse am besteu.

Beim Menschen sind die rein nervösen Störungen der MagenDarmmotilität, ein zu langes oder zu kurzes Verweilen der Ingesta im Verdauungsrohr, ausserordentlich häufig. Dass hier den zentralen nervösen Erregungen ein weitgehender Einfluss zukommt, ist durch zahlreiche klinische Beobachtungen erwiesen. Eine experimentelle Untersuchung dieser Verhältnisse im Tierversuch erschien wünschenswert.

Der Aufklärung bedurften vor allem die Fragen, wie die Erregung der genannten efferenten Nerven einwirkt auf die Verweildauer der Speisen im Magen, den Übergang vom Fundus zum Pylorus, die Reflexe und das rhythmische Spiel des Sphincter pylori, die Passage durch den Dünndarm, den Übertritt vom Dünndarm in den Dickdarm, die Weiterbeförderung im Dickdarm und die Defäkation.

\section{Methodik.}

Die Wahl der Methode bietet bei der Untersuchung der Innervation der Magen-Darmbewegungen durch efferente Nerven eine besondere Schwierigkeit. Auf die Einzelheiten der bisher gebräuchlichen Methoden soll nicht näher eingegangen werden. Sie sind von Magnus ${ }^{1}$ ) in Tigerstedts \#Handbuch der physiologischen

1) R. Magnus, Die Bewegungen des Verdauungsrohres. Handb. d. physiol. Methodik von R. Tigerstedt Bd. 2 Abt. 2 S. 99.1908. 
Methodik" eingehend kritisch besprochen. Es ist hervorzuheben, dass die meisten dieser Verfahren, wie die Anlegung einer Fistel in den Verdauungskanal uns nur über die Bewegungsänderungen in einem ziemlich eng begrenzten Abschnitt Auskunft zu geben vermögen. Auch die direkte Inspektion nach Eröffinung der Bauchhöhle, welche sehr starke Hemmungen bedingt, zeigt uns ohne weitere Eingriffe nur die oberfiächlichen Bewegungen, muss uns aber über andere wichtige Faktoren, wie z. B. die Funktion der Sphinkteren, im unklaren lassen.

Für die Untersuchung unserer Fragen scheint nur das $\mathrm{R}$ ö n t $\mathrm{g}$ e $\mathrm{n}$ verfahren in Betracht $z u$ kommen, das gestattet, die ganze Wanderung des Speisebreies durch den Verdauungstraktus von der Speiseröhre bis zum Rectum an demselben Tier zu beobachten, ohne dass man Eingriffe zu machen braucht, welche die Koordination der Magen-Darmbewegungen und der Magen-Darmreflexe zu stören imstande sind.

Schon Cannon ${ }^{1}$ ), der die Röntgenmethode zur Feststellung der normalen motorischen Vorgänge im Verdauungstraktus in die Pbysiologie einführte, suchte mit diesem Verfahren Aufschluss zu gewinnen über die zentralen Innervationsverhältnisse des Magens und Darms. Er durchschnitt bei einer Reihe seiner Versuchstiere, gewöhnlich Katzen, die beiden Vagi, in anderen Fällen die Splanchnici oder beide Nervengruppen zugleich und beobachtete am Röntgenschirm nach Ablauf der Operation an den nicht narkotisierten mit Wismutbrei gefütterten Tieren die Ausfallserscheinungen der Motilität des Magens und Darms. Er fand, dass alleinige Splanchnicusdurchschneidung die Magen-Darmbewegungen nicht verändert. Nach Vagusdurchschneidung kam es zu einer Schädigung der Magenperistaltik und einer Verzögerung des Speisetransportes durch Magen und Dünndarm. Viel geringer waren diese Schädigungen bei kombinierter Vagus- und Splanchnicusdurchschneidung. Die Pendelbewegungen res Dünndarms waren in allen seinen Fällen zu sehen.

Für das Studium der einfachen Nervendurchschneidung bietet die Cann on'sche Methore zweifellos die günstigsten physiologischen Bedingungen. Für eine Nervenreizung kann sie jedoch in dieser Form nicht in Betracht kommen. Bei der sehr grossen Abhängigkeit

1) W. B. Cannon, The motor activities of the stomach and small intestines after splanchnic and vagus section. Americ. journ. of physiol. vol. 17 p. 429.1906. 
der Magen-Darmmotilität von psychischen Erregungszuständen - Cannon sah immer dann, wenn die Tiere sehr unruhig wurden, Sistierung aller Magenbewegungen ${ }^{1}$ ) - würde der schmerzhafte Eingriff der Freilegung und Reizung der Nerven nur in Narkose geschehen können. Anderseits wirkt aber auch die tiefe Narkose nicht nur direkt auf die Magen-Darmbewegung [Jakobj $\left.\left.{ }^{2}\right)\right]$, sondern sie ist auch imstande, den Erfolg einer Nervenreizung abzusehwächen oder zu verändern.

Diese Überlegung führte dazu, in einer anderen Weise zu versuchen, Einflüsse psychischer Art auszuschalten und zwar durch direkte Entfernung des Gehirns. Eine geeignete Methode bot das Sherrington'sche Verfahren der Dekapitation ${ }^{3}$ ). Sherrington gelang es, durch Abtrennung des anämisch gemachten Kopfes mit Gehirn und Medulla olongata in tiefer Chloroformnarkose ein Präparat zu gewinnen, das nach Abklingen der Narkose bei künstlicher Atmung und Erwärmung 10 Stunden und mehr obne shockartige Nebenwirkungen, bei guter Herztätigkeit tadellose Reflexe zeigte. Die Einzelheiten des Verfahrens werden noch erörtert. An diesem dekapitierten Tier ist das Vaguszentrum mit der Medulla oblongata ausgesehaltet. Die ausserdem für den Magen-Darmkanal in Betracht kommenden Zentren des Sympathicus im Brust- und Lendenmark sowie die Ursprünge des sakralen autonomen Nervensystems des Pelvicus sind erhalten.

Wenn dieses Präparat einen geregelten Ablauf der Verdauungsbewegungen im Röntgenbild erkennen liess, schien es am meisten geeignet, den Einfluss der efferenten Nerven des Magen-Darmtraktus zu studieren. Einige Ausfallserscheinungen mussten freilich von vornherein zu erwarten sein, besonders weil die Vagusursprünge abgetrennt wurden. $O b$ dieser Ausfall der zentralen Vagusinnervation dann durch eine Reizung des peripheren Stumpfes ganz oder teilweise kompensiert werden kann, mussten die Versuche ergeben. Wir nehmen voraus, dass es in der Tat möglich war, dje dekapi-

1) W. B. Cannon, The influence of emotional states on the functions of the alimentary canal. The americ. journ. of the med. scienc. vol. 137 p. 480.1909.

2) C. Jakobj, Beiträge zur physiologischen und pharmakologischen Kenntnis der Darmbewegungen mit besonderer Berücksichtigung der Beziehung der Nebennieren zu denselben. Arch. f. exper. Path. u. Pharm. Bd. 29 S. 171. 1892.

3) C. S. Sherrington, A mammalian spinal preparation. Journ. of Physiol. vol. 38 p. 375.1909. 
Der Einfluss der Vagusreizung auf den Ablauf der Verdauungsbewegungen. 561

tierten Tiere bis zu $28 \mathrm{Stunden}$ bei relativ guter Herztätigkeit und guten Reflexen zu erhalten und dabei den Ablauf der Verdaungsbewegungen in allen Einzelheiten von der Magenfüllung bis zur Defäkation im Röntgenbild zu verfolgen.

Wie vermutet, zeigten die Verdauungsbewegungen an den Rückenmarkstieren gesetzmässige Abweichungen vom Normalen. Diese werden im folgenden sehr ausführlich geschildert, da sie die Grundlagen für die späteren Versuche über die Innervationsverhältuisse der MagenDarmbewegungen bilden.

\section{Versuchsanordnung.}

Als Versuchstiere dienten Katzen von mittlerer Grösse. Die Dekapitation geschah genau nach den Angaben Sherring ton's: "Der tief narkotisierten Katze wird eine Trachealkanüle angelegt; die Karotiden werden beiderseits unterbunden. Die Hant wird in der Höhe des Hinterhauptes, dicht hinter den Ohren quer durchgetrennt und rückwärts zurückgezogen, um die Halsmuskulatur am Epistropheus freizulegen. Dann sind die Enden der Transversalfortsätze des Atlas zu fühlen; gerade hinter diesen Fortsätzen macht man eine tiefe Inzision durch die Muskulatur. Der breite Prozessus spinosus des Epistropheus wird mit einer Knochenzange eingekerbt. Dicht unter dem Körper des Epistropheus wird mit einer scharfen Aneurysmennadel eine starke Ligatur hindurchgeführt und in der Rinne, welche durch die Inzision hinter den Querfortsätzen des Atlas und durch die Kerbe im Processus spinosus des Epistropheus gebildet wird, fest geknotet. Diese Ligatur komprimiert die Vertebralarterien, wo sie vom Querfortsatz des Epistropheus zum Querfortsatz des Atlas laufen. Eine zweite starke Ligatur wird dann in der Höhe des Krikoidknorpels um den Hals geschlungen und so gelegt, dass sie den ganzen Nacken mit Ausnahme der Trachea umfasst. Die Dekapitation wird mit einem Amputationsmesser vollendet. Sie geht von der ventralen Seite durch das Atlanto-Occipitalgelenk und durchschneidet das Rückenmark gerade hinter seiner Verbindung mit der Medulla oblongata. Die rund um den Hals gelegte Ligatur wird im Augenblick der Dekapitation fest zugezogen. Der abgeschnittene Kopf des tief narkotisierten Tieres wird dann entfernt. Die Blutung ist ausserordentlich gering. Wenn noch etwas Blut aus dem Wirbelkanal sickert, wird sie durch geringes Erheben des Halses über den übrigen Körper gestillt. . . . . . Die Hautlappen werden zusammengenäht, um das freiliegende Ende des Rückenmarkes und die anderen durch die Amputationswunde entblössten Gewebe zu bedecken." Die ganze Operation kann in 6 Minuten vollendet werden. In unseren Versuchen wurden bei der Karotidenunterbindung die Vagi beiderseits durchschnitten. Der dekapitierte Körper des Tieres liegt auf einem elektrisch erwärmten Operationsbrett und wird künstlich geatmet.

Wenige Minuten nach der Dekapitation kehren die Reflexe allmählich wieder, zuerst der Patellarreflex, dann der Schwanzreflex. Der Blutdruck ist 
bei diesen Tieren trotz vollständiger Entfernung der Medulla auffallend hoch. Nach Sherrington bewegt er sich um $80 \mathrm{~mm} \mathrm{Hg}$. In einem von mir angestellten Versuch betrug er 3 Stunden nach der Dekapitation $76 \mathrm{~mm} \mathrm{Hg}$. Herr Kollege Storm van Leeuwen teilte mir mit, dass er in sieben Versuchen Werte zwischen 54 und 70 , im Durchschnitt $64 \mathrm{~mm} \mathrm{Hg}$ fand.

Nachdem die Tiere 1-2 Stunden bei künstlicher Atmung und Erwärmung gelegen batten und man annehmen konnte, dass die Chloroformnarkose grösstenteils abgeklungen war, wurde durch eine Ösophagussonde der Wismutbrei in den Magen eingeführt.

Es erwies sich für unsere Zwecke deshalb als vorteilhaft, bei der zweiten Halsligatur ausser der Trachea auch den Ösophagus nicht mit abzubinden. Der Wismutbrei bestand aus $25 \mathrm{~g}$ Salzkartoffeln, $5 \mathrm{~g}$ Wismuthydrat und etwa $40 \mathrm{ccm}$ Wasser, zusammen zu einem feinen Mus verrieben. Wie spätere Versuche zeigten, machten geringere Schwankungen in der Konsistenz des Breies keinen Unterschied bei der Magenentleerung, wohl aber Differenzen in der Menge. Nach dieser Wismutfütterung wurde das Tier in das Röntgenzimmer gebracht, dort an die Leitung der künstlichen Atmung angeschlossen und konnte nun genau wie ein normales Tier zur Röntgendurchleuchtung beliebig oft aufgebunden werden. Die Wismutschatten auf dem Röntgenschirm wurden zu regelmässigen Zeiten auf Pauspapier aufgezeichnet.

Auf eine geregelte Erwärmung des Tieres war besonders zu achten, da stärkere Abkühlung oder Erhitzung augenscheinlich ausser den Reflexen auch die Verdauungsbewegungen veränderte.

Die Beobachtungszeit betrug meist 12-24 Stunden. Dann wurde das Tier durch Abstellen der künstlichen Atmung getötet. Manche Tiere wurden 26 Stunden und mehr am Leben gelassen, bis das Herz von selbst erlahmte. Fast in allen Fällen wurde der letzte Röntgınbefund durch die Sektion kontrolliert.

\section{Die Verdauungsbewegungen an der Rückenmarkskatze.}

Der Ablauf der Verdauungsbewegungen nach Ausschaltung des Gehirns und der Medulla oblongata wurde in elf Versuchen an der dekapitierten Katze beobachtet. Die Abweichungen vom normalen lebenden Tier, die hier zutage traten, betrafen vor allen Dingen die Tätigkeit des Magens. Wie aus den Untersuchungen von Cannon ${ }^{1}$ ) und von $\mathrm{Magnu \textrm {s } ^ { 2 }}$ ) hervorgeht, und wie ich mich selbst an einer Reihe von Versuchen überzeugen konnte, findet man bei der normalen Katze wenige Minuten nach der Fütterung mit Kartoffelbrei bereits Fundus und Pylorusteil des Magens gefüllt und den letztgenannten

1) Cannon, The movements of the stomach studied by means of the Röntgenrays. Americ. journ. of Physiol. vol. 1 p. 359. 1898.

2) Magnus, Die stopfende Wirkung des Morphins. II. Mitt. Pflüger's Arch. Bd. 122 S. 210.1908. 
Der Einfluss der Vagusreizung auf den Ablauf der Verdauungsbewegungen. 563

Teil schon in mehr oder weniger lebhafter peristaltischer Tätigkeit. Wesentlich anders verläuft dieser Vorgang bei der ihrer höheren Zentren beraubten Rückenmarkskatze. Kurz nach Einführung des
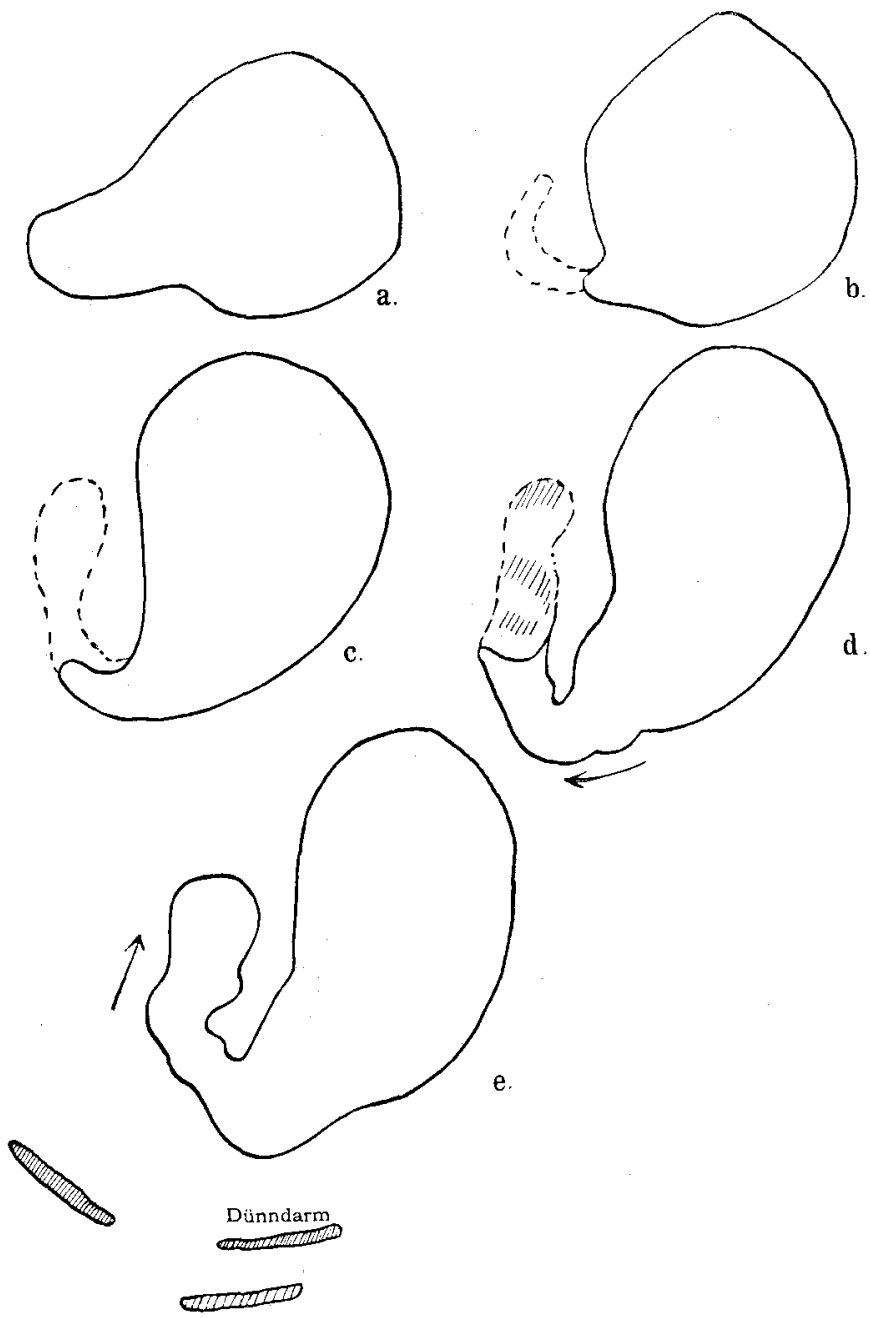

Fig. 1. $a$ Unmittelbar nach der Einführung des Wismutbreies. $b 10$ Minuten später. $c 25$ Minuten später. d 45 Minuten später, erstes Auftreten von Peristaltik. $\quad$ e 1 Stunde 10 Minuten später, erstes Auftreten der Dünndarmfüliung. (Auf $1 / 2$ verkleinert.)

oben beschriebenen Wismutbreies sieht man diesen entweder ganz im Fundus liegen oder höchstens Spuren davon im Pylorusteil. Der Fundus erscheint dabei bewegungslos und ziemlich voluminös. Irgend- 
welche Einbuchtungen, die auf eine Kontraktion schliessen liessen, fehlen vollkommen. Erst nach einer gewissen Zeit beginnt der Fundus eine andere Gestalt anzunehmen und den Pylorusteil mit Wismut zu füllen. Zugleich mit dem Auftreten des Wismuts im Pylorusteil sind dann auch zuerst leichtere, dann tiefere peristaltische Kontraktionswellen zu sehen. Die Zeit, die vergeht, bis man Pylorusteilfüllung und Peristaltik auftreten sieht, variiert sebr erheblich, zwischen 10 Minuten und $1^{1 / 2}$ Stunde, im Durchschnitt 45 Minuten nach der Fütterung (gegen 2-5 Minuten beim normalen Tier). Diesen langsamen verzögerten Übertritt des Wismutbreies lassen die vorstehenden Schirmpausen (Fig. 1) erkennen.

Nachdem die ersten peristaitischen Wellen erschienen sind, kann man sie regelmässig zum Pylorus hinlaufen sehen. Die einzelñen Wellen folgen sich in Zeitabständen von 10-15 Sekunden, Zahlen, die genau übereinstimmen mit den Befunden Cannon's an der normalen Katze. Diese uhrmässige Regelmässigkeit der Pylorus. peristaltik ist also in keiner Weise durch die Dekapitation gestört. Die Kontraktionen erscheinen im Anfang etwas oberflächlich. Doch nehmen sie langsam an Tiefe zu und unterscheiden sich dann kaum noch von normalen Kontraktionen. Sie lassen sich beobachten, solange noch Wismutbrei im Pylorusteil des Magens nachzuweisen ist.

Der Beginn des ersten im Röntgenbild nachweisbaren Übertrittes von Wismut-Kartoffelbrei aus dem Magen in das Duodenum findet bei der normalen Katze durchschnittlich nach 20 Minuten statt. Bei der dekapitierten Katze ist dieser erste Übertritt ausserordentlich stark verzögert. Über die bei den einzelnen Versuchen festgestellten zeitlichen Verhältnisse gibt die nachstehende Tabelle Auskunft (Tab. I).

Tabelle I.

\begin{tabular}{|c|c|c|c|c|c|c|c|c|c|c|c|c|}
\hline Versuch & 1 & 2 & 3 & 4 & 5 & 6 & 7 & 8 & 9 & 10 & 11 & Mittel \\
\hline $\begin{array}{l}\text { Zeitintervall zwischen De- } \\
\text { kapitation und Fütte- } \\
\text { rung in Stunden . } \\
\text { Füllung und Peristaltik } \\
\text { des Pylorusteils. (Stun- } \\
\text { den nach Fütterung.) } \\
\text { Erstes Sichtburwerdendes } \\
\text { Wismuts im Dünndarm. } \\
\text { (Stunden n. Fütterung.) }\end{array}$ & $1 / 4$ & $3 / 4$ & $3 / 4$ & 2 & $1^{1 / 4}$ & $1^{1 / 4}$ & $1^{1 / 4}$ & $1^{1 / 2}$ & $2^{1 / 2}$ & $2^{3 / 4}$ & $10^{\prime}$ & $1^{3 / 4}$ \\
\hline
\end{tabular}


Erst nach $1^{3 / 4}$ Stunden im Mittel lässt sich Dünndarmfüllung nachweisen. Selust in den Fällen, in welchen schon 10-15 Minuten nach Fütterung gute Pylorusperistaltik auftrat, sah man die Wellen oft über 1 Stunde regelmässig zum Pylorus laufen, ohne dass sich sein Sphinkter öffnete. Die starke Verspätung bei Versuch 1 ist vielleicht auf eine im Anfang bestehende Übererwärmung des Tieres zurückzuführen, die auch für mehrere Stunden eine starke Beeinträchtigung der äusseren Reflexe veranlasste. Aber auch abgesehen von diesem Versuche finden sich noch Schwankungen zwischen 1 und $2^{1 / 2}$ Stunden, für die aus der Versuchstechnik keine Erklärung zu gewinnen ist. Jedenfalls kann man den frühesten Beginn der Magenentleerung bei der dekapitierten Katze erst 1 Stunde nach der Fütterung erwarten. Geringere Unterschiede der Zeitdauer zwischen Dekapitation und Fütterung üben, wie aus einem Vergleich mit der ersten Rubrik der Tabelle hervorgeht, keinen nachweisbaren Einfluss auf den Beginn der Magenentleerung aus.

Füttert man eine normale Katze mit Wismut-Kartoffelbrei in der von uns gebrauchten Quantität, so ist nach $3-4$ Stunden der Magen leer. Die Entleerung vollzieht sich anscheinend in der Weise, dass der Fundus seinen Inhalt gleichmässig in den Pylorusteil presst, wo er durch peristaltische Wellen in das Duodenum befördert wird (Cannon). Eine anatomisch bestimmbare Grenze dieser beiden Funktionen der Magenmotilität kann dabei wohl kaum gegeben werden. Es scheint vielmehr ein allmählicher Übergang zu bestehen. Die peristaltischen Wellen greifen jedenfalls über das transversale Band oder den sogenannten Sphincter antri pylori hinaus.

Anders verlaufen diese Vorgänge an der dekapitierten Katze. In keinem Falle wurde eine völlige Entleerung des Magens beobachtet. Er entleert sich nur bis zu einem gewissen Grade, und zwar liegt der Grund offenbar in dem Unvermögen des Fundusteiles, seine tonische Kontraktion über eine bestimmte Grenze hinaus noch weiter zu steigern. Man sieht nämlich $7-8$ Stunden lang regelmässige Pylorusteilperistaltik, dann hört sie allmählich auf, der Pylorusteil erscheint völlig entleert und nur in dem kugelförmig aussehenden, bewegungslosen Fundus bleibt noch ein grosser Teil des Wismutbreies liegen. Ein weiterer Übergang von Wismutbrei in den motorisch tätigeren Pylorusteil findet nicht mehr statt, es sei denn, dass man durch neue Fütterung den Magen auf seinen früheren Füllungszustand bringt. Manchmal gelingt es, durch sanften Fingerdruck wieder Wismutbrei in den Pylorusteil zu pressen.

Zur Veranschaulichung dieser Verhältnisse dienen umstehende Abbildungen (Fig. 2). Fig. 2 a zeigt das Röntgenbild des Magens einer normalen Katze 3 Stunden nach des Wismutmahlzeit, Fig. 2 b von einer dekapitierten Katze 10 Stunden nach 
der Wismutmahlzeit. Bei dem Normalversuch war in der nächsten Stunde der ohnehin nur sehr undeutliche Magenschatten vollkommen rerschwunden, bei der dekapitierten Katze blieb er unverändert.

Nicbt obne Interesse scheint es, dass sich bei zwei an Pneumonie erkrankten Katzen ähnliche Speiseretentionen im Magenfundus feststellen liessen, wie wir sie bei der Rückenwarkskatze als Regel fanden.

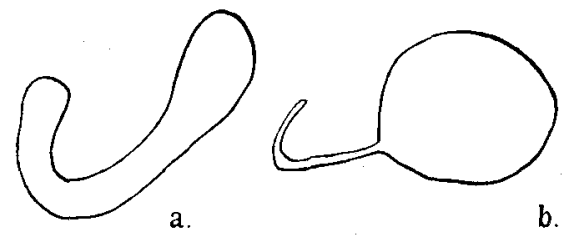

Fig. 2. (Auf $1 / 2$ verkleinert.)

Wie Cann on am Röntgenschirm beobachtete, entleert sich der Magen nach der ersten öffnung des Pylorus in regelmässigen Güssen in das Duodenum. Diese Regelmässigkeit der Pylorusöffnung ist am dekapitierten Tier nicht festzustellen. Man beobachtet, wie schon oben erwähnt, zwar auch hier eine regelmässige peristaltische Tätigkeit, doch ist der Übertritt durch den Sphinkter in das Duodenum sichtlich gestört. Die einzelnen Ergüsse sind seltener und auch unregelmässiger. Man sieht längere Zeit lindureh lebhafte peristaltische Magentätigkeit, ohne dass sich der Dünndarm weiter füllt. Es ist also nicht allein die erste Öffnung des Pylorus abnorm verzögert, sondern auch seine weitere rhythmische Öfnung und Schliessung gegenüber dem normalen gestört.

Was nun die Bewegungen des Dünndarmes selbst angeht, so kann man die verschiedenen Arten dieser Bewegungen, die rhythmischen Segmentierungen $\left.\left[\mathrm{Cann}_{0} \mathrm{n}^{1}\right)\right]$ oder Pendelbewegungen (L ud wig) und die Peristaltik auch am Rückenmarkstier in deutlicher Weise verfolgen. Soweit es sich mit dem Röntgenschirm feststellen lässt, ist hier ein deutlicher Unterschied zwischen dem normalen und dem dekapitierten Tier nicht zu bemerken. Ebenso wie am lebenden Tier sind die peristaltischen Bewegungen am stärksten ausgeprägt in dem duodenalen Teil des Dünndarms. Hier betrug in einigen Fällen die Geschwindigkeit der peristaltischen Welle $3 \mathrm{~cm}$ und mehr in der Minute. In den distaleren Gebieten des Dünndarms war die Peristaltik jedoch erheblich langsamer, besonders im letzten Drittel, wo sich der Wismutbrei oft in dicken Haufen, stark eingedickt (Sektion) ansammelte.

1) Cannon, The movements of the intestine studied by means of the Röntgenrays. Americ. journ. of Physiol. vol. 6 p. 251. 1902. 
Nur in einem Falle trat einmal eine sehr stark beschleunigte peristaltische Welle auf, die einen aus dem Magen ausgetretenen Wismutstreifen von $7 \mathrm{~cm}$ Länge in wenigen Minuten in den Dickdarm beförderte. Es erinnerte diese starke peristaltische Welle an die von $\mathrm{v}$. Braam-Houkgeest ${ }^{1}$ ) postmortal beobachteten Rollbewegungen oder peristaltic rush von Meltzer und Auer ${ }^{2}$ ). Irgendwelche Beschleunigung der Magen- oder Dickdarmmotilität bestand in diesem Falle nicht. Auch die Sektion ergab nichts Besonderes.

Die rhythmischen Segmentierungen sah man in allen Teilen des Dünndarms gleichmässig, sehr schön und lebhaft oft schon einige Sekunden nach dem Austreten des Wismutbreies aus dem Magen. Im unteren Abschnitt des Dünndarms erschienen die Segmente meist etwas voluminöser, vielleicht entsprechend der stärkeren Eindickung.

Im Zusammenhang mit dem kaum vom Normalen abweichenden Verhalten der Dünndarmmotilität ist auch der Beginn des ersten sichtbaren Auftretens von Wismutbrei im Colon nicht sehr verzögert. Im Mittel fand ich als Zeitdifferenz zwischen dem ersten Sichtbarwerden des Wismutbreies im Duodenum und dem im Colon Schwankungen von $1 \frac{1}{2}-4$ Stunden (im Mittel 21/4 Stunden) bei sechs Versuchen am normalen Tier. In zehn Versuchen an der dekapitierten Katze betrug dieser Zeitunterschied ebenfalls $1^{1 / 2}-4$ Stunden (im Mittel $3^{1_{1}}$ Stunden). Im Verhältnis zu den viel stärkeren Störungen der Magenmotilität ist diese Verspätung relativ gering.

Auch die Bewegungen des Dickdarms waren am dekapitierten Tier mit mehr oder weniger grosser Regelmässigkeit zu verfolgen. Die Antiperistaltik des proximalen Teiles des Colons war in einigen Fällen sehr deutlich und von normaler Schnelligkeit und Tiefe der Kontraktionen. In anderen Fällen war sie nur oberflächlich oder fehlte ganz. Doch ist diese Art von Darmbewegung auch am normalen Tier nicht immer mit derselben Gleichmässigkeit zu sehen (Ca n n o n). Fand zur Zeit der Durchleuchtung gerade Übertritt eines Teiles des Dünndarminhaltes in den Dickdarm statt, liess sich auch die von Cannon beschriebene starke Kontraktion des proximalen Colons in der Höhe des Sphincter ileocolicus in ausgeprägtester Weise be-

1) v. Braam-Houkgeest, Untersuchungen über die Peristaltik des Magenund Darmkanals. Pflüger's Arch. Bd. 6 S. 266. 1872. Zit. nach Magnus, Die Bewegungen des Verdauungsrohres. Tigerstedt's Handb. d. physiol. Methodik.

2) Meltzer and Auer, Peristaltic rush. Americ. journ. of Physiol. vol. 20 p. 259. 1907.

Pflüger's Archiv für Physiologie. Bd. 145. 
obachten. Diese Kontraktion löste sịch nach kurzer Zeit wieder, und es folgten antiperistaltische Wellen. An der Grenze zwischen proximalem und distalem Colon befand sich in den meisten Fällen eine mehr oder minder starke Einschnürung. Über diesen "Kontraktionsring " hinaus waren nie antiperistaltische Wellen nachzuweisen. Überhaupt waren in diesem letzten Drittel des Dickdarmes nur selten motorische Veränderungen sichtbar. Wie beim normalen Tier, bestanden auch hier die Bewegungen hauptsächlich in tonischen Kontraktionen, welche den eingedickten Kot in Ballen abschnürten und von Zeit zu Zeit langsam dem Rectum zuführten. Bei der beschränkten Beobachtungszeit war die Defäkation von wismuthaltigem Stuhl nur selten zu sehen., Mehrmals liess sich eine solche durch den Reiz des in den After eingeführten Thermometers reflektorisch erzielen. Der Kot wurde dann etwa von der Mitte des distalen Colons an durch eine langsame peristaltische Kontraktion durch den erschlaffenden Sphincter ani hinausgepresst. Auch beim dekapitierten Tier war dieser Sphinkter sonst immer tonisch geschlossen. Der Übergang des Dickdarminhaltes vom proximalen zum distalen Colon trat ziemlich regelmässig durchschnittlich $2-3$ Stunden nach der ersten Colonfüllung auf, eine Zeitdauer, die auch beim lebenden Tier als Normalzeit gelten kann, wie mich sechs Versuche belehrten.

Wir können die geschilderten Ergebnisse folgendermaassen zusammenfassen: Nach Abtrennung von Gehirn und Medulla oblongata lassen sich an der künstlich geatmeten und erwärmten Rückenmarkskatze, bei der also eine Beeinflussung der Verdauungsbewegungen durch das Vaguszentrum nicht mehr stattfindet, alle vom normalen lebenden Tier her bekannten Arten von Bewegungen des Magen-Darmkanals mittels des Röntgenverfahrens studieren. Keine wesentlichen Abweichungen von normalen zeigen die rhythmischen Segmentierungen und die peristaltische Tätigkeit des Dünndarms, die Antiperistaltik und die tonischen und peristaltischen Kontraktionen des Dickdarms. Nicht gestört ist die Dauer des Übertrittes des Wismutkotes vom proximalen zum distalen Colon und die Defäkation. Sehr wenig verzögert ist die Passage des Speisebreies durch den Dünndarm. Erheblichere Abweichungen vom Normalen bietet die Motilität des Magens. Der Übergang des gefütterten Wismutbreies vom Fundus in den Pylorusteil ist stark verzögert. Auch die Peristaltik des Pylorusteiles tritt verspätet auf. Das rhythmische Spiel des Sphineter pylori ist unregelmässig, die Öffnung des Sphinkters seltener als 
normal. Eine völlige Entleerung des Fundus findet nicht statt, während sich der Pylorusteil des Magens zwar verlangsamt, aber doch vollständig entleert.

Diese Resultate an der Rückenmarkskatze zeigen eine weitgehende Übereinstimmung mit den Ergebnissen Cannon's am lebenden vagotomierten Tier.

b) Die Verdaungs bewegungen an der Rü ckenmarkskatze nach Durchschneidung der oberen Nn. splanchnici.

Nach den Befunden von $\mathrm{Cann}$ on und anderen Autoren ist die Tätigkeit des Magens nach beiderseitiger Vagotomie weniger geschädigt, wenn man die hemmenden Splanchnici durchschneidet. Auch bei der dekapitierten Katze war ein derartiger hemmender Einfluss auf sympathischem Wege wahrscheinlich. Es wurden deshalb an sechs Katzen vor der Dekapitation beiderseits in tiefer Chloroformnarkose die Splanchnici majores, in einem Falle auch die minores durchschnitten.

Um durch Eröffnung der Bauchhöhle keine störenden Reize zu verursachen, wurde mit einigen $\mathrm{Abänderungen} \mathrm{das} \mathrm{von} \mathrm{Asp}$ angegebene Verfahren der retroperitonealen Splanchnicotomie angewandt. Man geht an der Grenze der langen Rückenmuskeln und der queren Bauchmuskeln dicht unter der letzten Rippe in die Tiefe, verfolgt die Vena lumbalis I bis zur Nebenniere, schiebt diese mit einem Finder etwas zur Seite und sieht dann in dem suprarenalen Fettgewebe das Ganglion coeliacum, zu dem der Splanchnicus major verläuft. Der Nerv wird mit einem Häkchen angehakt und durchschnitten. Die Anklemmung der Nebenniere oder Abbindung der $\mathrm{A}$. und $\mathrm{V}$. lumbalis ist nicht notwendig. Der Nerv liegt links meist tief in dem Zwerchfellwinkel; rechts muss die Leber etwas nach oben geschoben werden. Nach Vernähung der Hautwunde wird die Dekapitation nach Sh err in g to o direkt angeschlossen. Die ganze Operation kann in 20 Minuten vollendet werden.

In der Tat fand sich auch bei der dekapitierten Katze eine zweifellose Verbesserung der Magenmotilität nach Splanchnicotomie. Der Übergang des Wismutbreies aus dem Fundus in den Pylorusteil war entschieden erleichtert. Während bei erbaltenen Nn. splanchnici dieser Vorgang bis zu $1 \frac{1 / 2}{2}$ Stunde verzögert war, fand sich in allen Fällen, in denen diese Nerven durehsehnitten waren, spätestens nach 1/2 Stunde vollkommene Füllung und sehr deutliche Peristaltik des Pylorusabschnittes des Magens. Ein längeres bewegungsloses Verharren des Inhaltes im Fundusteil war niemals zu beobachten. Am auf- 
fallendsten war jedoch die Art der Peristaltik selbst verändert. Die Wellen liefen nicht allein mit ausserordentlich grosser Regelmässigkeit zum Pylorus, sondern zeigten auch so tiefe Kontraktionen, wie sie selbst beim normalen lebenden Tier selten beobachtet werden konnten. Diese so ausgeprägte Pylorusperistaltik wurde in keinem Falle vermisst.

Ferner verriet sich auch der Wegfall von Hemmungen durch eine Beschleunigung der ersten Magenentleerung. Nach 45 Minuten im Durchschnitt begann die Dünndarmfüllung. Bei erhaltenen Nn. splanchnici betrug diese Zeit $1^{3 / 4}$ Stunde.

\section{Tabelle II}

Dekapitation + Splanchnicotomie.

\begin{tabular}{c|c|c|c|c|c|c|c}
\hline Versuch & 1 & 2 & 3 & 4 & 5 & 6 & Mittel \\
\hline $\begin{array}{c}\text { Erstes Sichtbarwerden des Wismut. } \\
\text { breies im Dündarm. (Stunden } \\
\text { nach Fütterung)....... }\end{array}$ & $1 / 2$ & $11 / 2$ & $3 / 4$ & $20^{\circ}$ & 1 & $3 / 4$ & $3 / 4$
\end{tabular}

Nur in einem Versuch (Nr. 2 der Tabelle) war die erste Magenentleerung bis $1 \frac{1}{2}$ Stunde verzögert. Dieser Fall unterschied sich dadurch von den anderen, dass die Splanchnicotomie nicht vor, der Dekapitation in tiefer Chloroformnarkose vorgenommen wurde, sondern nach der Dekapitation. Die Narkose war schon abgestellt, und es machten sich bei der Präparation der Splanchnici sehr lebhafte Reflexe bemerkbar. Ausserdem wurde bei der Operation die linke Nebenniere stark beschädigt. $O b$ diese technischen Unvollkommenheiten ursächlich mit der gegenüber den sonstigen Befunden verzögerten Magenentleerung im Zusammenhang stehen, ist zwar möglich, aber nicht bewiesen. Beachtenswert erscheint, dass bei dem einzigen Versuch, in dem neben dem Splanchnicus major auch die minores vollständig durchschnitten waren, die erste sichtbare Pylorusöffnung am frühesten eintrat, schon nach 20 Minuten (Versuch 4). Irgendwelche sicheren Schlüsse, etwa auf eine erweiterte Ausschaltung von Hemmungsfasern, kann man jedoch aus diesem einzigen Falle nicht ziehen.

Nach der Splanchnicotomie ist ausser der verstärkten Pylorusperistaltik und der beschleunigten ersten Magenöffnung auch die Menge des in der Zeiteinheit in den Dünndarm beförderten Speisebreies vergrössert. Die nachstehende Kurve 1 wird diese Verhältnisse am besten illustrieren.

Als Maass für die Dünndarmfüllung ist die Länge der Wismutdünndarmschatten in Zentimetern angegeben, eine Art der Darstellung, die von Cannon zuerst verwandt wurde. Die Dünndarmschatten sind stündlich gemessen. Man 
erkennt, wie am normalen Tier die Füllung des Dünndarms sehr schnell zunimmt, nach 3 Stunden ihren Höhepunkt erreicht und dann wieder ziemlich steil abfällt. Beim dekapitierten Tier ohne Splanchnicotomie beginnt die Dünndarmfüllung erst nach langer Verspätung, steigt dann sehr langsam an und erreicht nicht einmal die Hälfte der Höhe der normalen Füllung. Viel mehr nähert sich dem normalen die Dünndarmfüllung nach Dekapitation und Splanchnicotomie. Sie beginnt nur wenig verspätet und erreicht ziemlich bald eine erheblich höhere Menge als ohne Splanchnicotomie.

Eine völlige Magenentleerung war auch nach Durchschneidnng der Splanchnici majores nicht zu erzielen. Auch hier fand sich selbst nach langer Beobachtungszeit (bis zu 26 Stunden) immer eine Speiseretention. In ähnlicher Weise wie bei reiner Dekapitation fand sich dann meist der Pylorusteil leer und der Fundus noch ziemlich

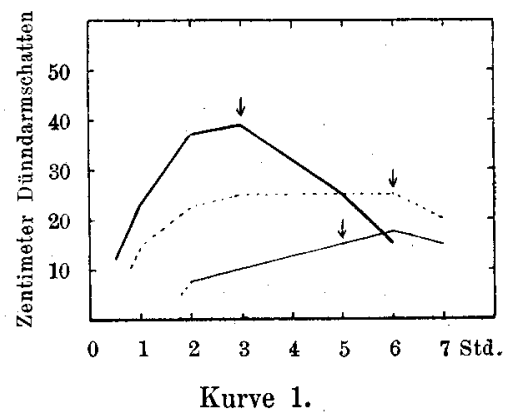

- Normaltier.

… Dekapitation mit Splanchnicotomie. $\downarrow$ Beginn " der Dickdarmfüllung. deutlich gefullt.

Die Bewegungsarten des Dünndarms unterschieden sich nach Splanchnicotomie nicht besonders von den oben geschilderten. Peristaltik und rhythmische Segmentationen waren unverändert. Rollbewegungen konnten wicht beobachtet werden. Irgendwelche Beschleunigung der Passage des Wismutbreies durch den Dündarm fand nicht statt. Im Gegenteil, es erschien fast, als ob in dem unteren Drittel des Dünndarms eher eine Verlangsamung der Fortbewegung einträte, als ob sich der Inhalt in stärkerem Maasse vor der lleocoecalklappe anstaue. In der Tat war bei den fünf Versuchen ein verlangsamter und verspäteter Eintritt der sichtbaren Colonfüllung unverkennbar. Bei erhaltenen Splanchnici majores dauerte die Passage des ersten Wismutballens vom Pylorus zum Coecum etwa 3 Stunden. Als Mittel bei fünf Versuchen bei Splanchnicusdurchschneidung fanden wir eine Durchgangszeit von 6 Stunden bis zum ersten Auftreten im Colon. Nur in einem Falle erfolgte dieser Übertritt schneller, nämlich 4 Stunden nach der ersten Magenentleerung. Es war dies der Versuch, in dem auch die Splanchnici minores durchschnitten waren.

Die splanchnischen Äste, die zum Colon laufen, d. h. die durch das Ganglion mesentericum inferius gehenden Splanchnici inferiores 
waren immer erhalten. Somit war ein beschleunigender Einfluss auf die Bewegungen des Dickdarms nicht zu erwarten. Unsere Versuche lassen sogar eher auf eine Verlangsamung der Fortbewegung im Dickdarm schliessen. Das Coecum füllte sich auffallend langsam. Der Wismutbrei ging nur in einem einzigen Falle über die Grenze des proximalen Colons hinaus und da nur in Spuren, wie bei der Sektion festgestellt wurde. Meist fand eine starke Ansammlung des Wismutkotes im proximalen Colon und im Coecum statt. Die Bewegungsverhältnisse im distalen Colon sowie die Defäkation konnten deshalb hier nie beobachtet werden.

Über das Vorhandensein von antiperistaltischen Wellen im proximalen Teil des Colons ist in keinem Protokoll etwas vermerkt, obwohl sorgfältig darauf geachtet wurde.

Bei Einübung der Technik gelang im Anfang die beiderseitige Durchschneidung manchmal nicht, und es wurde nur einer der beiden Splanchnici majores durchtrennt, viermal nur der rechte, einmal nur der linke. Ganz regelmässig war dann bei diesen Versuchen der Ablauf der Magen-Darmbewegungen sehr stark gehemmt. Nur Spuren Wismut traten in den Dünndarm über und dann erst nach sehr langer Zeit. Deutliche Pylorusperistaltik trat kaum auf. Manchmal entleerte sich der Magen gar nicht. Bis in den Dickdarm kamen die geringen Wismutmengen nicht. Die Hemmung der Bewegungen des Magens und Dünndarms waren so erheblich, dass man nach den ersten Durchleuchtungen ohne weiteres sagen konnte, ob es gelungen war, beide Splanchnicizu durchschneiden, oder ob man nur einen durchtrennt hatte. Diese Hemmung war auch viel stärker, als dann, wenn beide Splanchnici erhalten blieben.

Es ergibt sich aus diesen Versuchen, dass am dekapitierteu Tier doppelseitige Durchschneidung der Splanchnici majores die Magentleerung beschleunigt. Die Pylorusfüllung und Pylorusperistaltik tritt schneller auf. Die einzelnen peristaltischen Wellen sind kräftiger. Der erste Übertritt von Wismutbrei in das Duodenum nähert sich mehr der normalen Zeit. Die Menge des in den Dünndarm beförderten Mageninhaltes ist grösser. Die Bewegungsarten des Dünndarms sind unverändert. Der Übertritt von Wismutbrei in den Dickdarm ist verlangsamt, ebenso die Fortbewegung des Kotes im Dickdarm.

\section{Der Einfluss der Vagusreizung auf den Ablauf der Verdaungsbewegungen.}

Dass durch Reizung des Vagus Bewegungen und Bewegungsänderungen am Magen-Darmkanal auftreten, ist dureh eine Reihe 
von Arbeiten bewiesen. Jakobj ${ }^{1}$ ) untersuchte diese Verbältnisse bei Kaninchen und Katzen, die 3-6 Tage gehungert hatten, nach Eröffnung der Bauchhöhle, im Kochsalzbad. Auf Reizung eines Vagus am Halse erhielt er starke Bewegungen des Magenfundus und starke Darmperistaltik. Dieser Effekt trat jedoch nur ein, wenn vorher die Splanchnici durehsehnitten waren. Die bessere Wirkung der Vagusreizung nach Durchtrennung der hemmenden sympathischen Fasern betonen auch Bayliss und Starling ${ }^{2}$ ). Beim Hunde fanden diese Autoren nach Vagusreizung eine kurz dauernde Hemmung und darauffolgende Verstärkung der rhythmischen Kontraktionen des Darmes. Nach $\mathrm{Page} \mathrm{May}^{3}$ ) enthält der Vagus erregende und hemmende Fasern. Die Hemmung dauert manchmal während der ganzen Periode der Reizung an, manchmal nur 10-15 Sekunden, und es folgen verstärkte Kontraktionen zugleich mit einem Anwachsen des Tonus. Ähnliche Verhältnisse fand er an der Cardia und am Pylorus. Anfänglicher Erschlaffung folgte eine Kontraktion der Sphinkteren. Nach $O$ penchow ski ${ }^{4}$ ) ruft Vagusreizung Schluss des Pylorus hervor.

$\mathrm{Ob}$ der Dickdarm Nervenfasern vom Vagus erhält, ist bei der Katze zweifelhaft. Meltzer und Auer ${ }^{5}$ ) erhielten nach Vagusreizung beim Kaninchen Coecumkontraktionen. Langley ${ }^{6}$ ) vermutet, dass die Vagusfasern auch noch das proximale Colon innervieren. Nach Bayliss und Starling ${ }^{7}$ ) laufen nur sympathische und sakral autonome Nerven (Nn. pelvici) zum Colon.

1) Jakobj, l. c. (Dort ist anch die ältere Literatur über die Wirkung des Vagus auf Magen- und Darmbewegung angeführt.)

2) Bayliss and Starling, The movements and innervation of the small intestine. Part I. Journ. of Physiol. vol. 24 p. 99. 1899. Parts II and III. Journ. of Phys. vol. 26 p. 126. 1901.

3) Page May, The innervation of the sphincters and musculature of the stomach. Journ. of Physiol. vol. 31 p. 260. 1902.

4) Openchowski, Über die nervösen Vorrichtungen des Magens. Zentralbl. f. Physiol. Bd. 3 S. 1. 1889. Zit. nach Starling, Überblick über den gegenwärtigen Stand der Kenntnisse über die Bewegungen und die Innervation des Verdauungskanals. Ergebn. d. Physiol. Bd. 1 (2) p. 446. 1902.

5) Meltzer and Auer, Proc. Soc. Exper. Biol. New York 1907 no. 4 p. 39. Zit. nach Cannon, The mechanical factors of digestion. (Arnold, London 1911.)

6) Langley, Das sympathische und verwandte nervöse System der Wirbeltiere. Ergebn. d. Physiol. Bd. 2 (2) S. 818. 1908.

7) Bayliss and Starling, The movements and innervation of the large intestine. Journ. of Physiol. vol. 26 p. 107. 1900. 
Meine Beobachtungen über den Einfluss der elektrischen Reizung des peripheren Vagusstumpfes auf die Verdauungsbewegungen an der Rückenmarkskatze gründen sich auf Versuche an 29 dekapitierten Katzen. Bei fünf von diesen Tieren wurde unter verschiedenen Verhältnissen speziell der Dickdarm untersucht. Auf die Versuchsanordnung in diesen letzteren Fällen wird noch bei Besprechung des Dickdarms eingegangen werden.

In den übrigen Experimenten abber die Vaguswirkung auf den gesamten Ablauf der Verdauungsbewegungen war die Versuchsanordnung folgende:

Dekapitation und Fütterung erfolgten in der bereits oben geschilderten Weise. Nachdem man sich durch einige Durchleuchtungen versichert hatte, dass die Magenbewegungen sich nicht abnorm von den oben als normal befundenen unterschieden, wurden die Vagi am Halse frei präpariert, mit einem Faden angeschlungen und je auf ein Platinelektrodenpaar gelegt. In den ersten Versuchen wurden die Vagi bereits bei der Dekapitation frei präpariert, angeschlungen und einige Male auch sofort auf Elektroden gelegt. Es stellte sich jedoch heraus, dass dieses Verfahren nicht zweckmässig war, da augenscheinlich durch diese Manipulationen an den Nerven schon abnorme Reize gesetzt wurden, welche die Magenentleerung gegenüber den Befunden bei einfacher glatter Vagusdurchschneidung zu beschleunigen imstande waren, wie eine Reihe von Kontrollversuchen ergab.

Als sehr geeignete Elektroden erwiesen sich die von Sherrington angegebenen T-förmigen Glasröhrenelektroden, die ein Austrocknen und eine Kompression des Nerven verhindern. Diese Elektrodenpaare wurden beiderseits neben die Trachealkanüle gebettet und die Haut darüber mit einer Klemme geschlossen. Blieb das Tier längere Zeit auf dem Durchleuchtungstisch liegen, was bei den Reizversuchen meist vorteilhaft war, erfolgte die Erwärmung durch eine elektrische Tischlampe von oben.

Als Stromquelle diente ein Akkumulator, der mit einem nach Kronecker geeichten Schlittenapparat mit 10000 Windungen verbunden war. Die Eichungsskala geht von 1-10000.

Es wurden immer beide Vagi zugleich gereizt, da nach den Befunden Jakobj's manchmal der rechte Vagus mehr auf den Magen und der linke mehr auf den Darm wirkt oder umgekehrt. Die Zeitdauer der einzelnen Reizungen betrug selten mehr als $1^{1 / 2}$ Minuten. Die Länge der Pausen zwischen den verschiedenen einzelnen Reizungen wechselte.

Bevor die Vagi gereizt wurden, überzeugte man sich durch verschiedene Durchleuchtungen, dass die Anlage der Elektroden usw. die normalen Bewegungen nicht wesentlich verändert hatte.

Die Beobachtung wurde gewöhnlich so ausgeführt, dass man erst eine gewisse Zeit durchleuchtete, dann während der Durchleuchtung etwa 1 Minute reizte, und dann nach Suhluss der Reizung noch einige Minuten weiter durchleuchtete. 
Die angewandte Stromstärke schwankte zwischen 40 und 1000 Einheiten des Kronecker'schen Schlittens, von denen also, da ja beide Vagi zugleich gereizt wurden, auf einen Vagus nur die Hälfte kommt. In einigen Fällen war schon bei Stromstärken zwisehen 17 und 40 ein deutlicher Effekt auf Magen und Darm zu erhalten. Die deutlichste Vaguswirkung auf den Magen trat meist bei einer Reizstärke auf, die den Puls etwa auf die Hälfte verlangsamte. Es liess sich jedoch auch in einigen Fällen einwandfrei zeigen, dass schon stärkere Veränderungen der Magen-Darmbewegungen sichtbar wurden bei Reizen, die noch nicht merkbar auf die Pulsfrequenz einwirkten. Stromstärken, die den Puls sehr hochgradig verlangsamten oder Herzstillstand erzeugten, wurden nur selten angewandt.

Da die Herzwirknng der Vagusreizung in den einzelnen Versuchen bald bei geringerem, bald bei grösserem Rollenabstand eintrat, wird bei Schilderung der Resultate als Maassstab für die Reizstärke nicht der Rollenabstand des Kronecker'schen Schlittens, sondern die Grösse der Pulsverlangsamung angegeben. Ströme, die keine merkbare oder nur minimale Pulsverlangsamung machten, sind als schwache Ströme bezeichnet, solche, die den Puls etwa um die Hälfte verlangsamten, als mittlere, und solche die zu hochgradiger Pulsverlangsamung oder Herzstillstand führten, als starke Ströme. Diese Unterscheidung ist nötig, da, wie die Versuche zeigten, die Art der Bewegungsänderung des Magens und Darms auf Vagusreizung sehr verschieden sein kann, je nachdem stärkere oder schwächere Ströme verwandt werden.

Die Wirkung der Vagusreizung auf den Blutdruck der dekapitierten Katze wurde in einem besonderen Versuche bestimmt. Der Blutdruck in der Karotis betrug hier 3 Stunden nach der Dekapitation $76 \mathrm{~mm} \mathrm{Hg}$. Eine Stromstärke von 40 Einheiten des Kronecker'scheu Schlittens liessen Pulsfrequenz und Blutdruck unverändert. Eine Stromstärke von 250 Einheiten verlangsamte den Puls auf die Hälfte und liess den Blutdruck auf etwa $50 \mathrm{~mm} \mathrm{Hg.}$ sinken.

Diese infolge von Vagusreizung auftretende Zirkulationsstörung ist jedoch nicht die Ursache der Bewegungsänderungen am Magen und Darm. Denn es können nach Vagusreizung oft sehr heftige Verdauungsbewegungen auftreten, ohne dass bei dieser Stromstärke schon eine erkennbare Kreislaufstörung entsteht. Auch die bei mittlerer Stromstärke auftretende Blutdrucksenkung genügt nicht, 
um die Magendarmwirkung des Vagus zu erklären. Denn diese Blutdrucksenkung ist nicht grösser als die, welche bei Splanchnicusdurchschneidung eintritt; und bei Splanchnicusdurchschneidung treten derartige heftige Verdauungsbewegungen wie bei Vagusreizung mit mittleren Strömen nie auf. Diskutabel erscheint bloss die Frage, ob die bei mittleren Strömen auftretende schnell vorübergehende Hemmung der Magenperistaltik nicht auf den plötzlichen Blutdrucksturz zurückzuführen ist. Auch diese Ansicht ist unwahrscheinlich nach den Versuchen von Page May, der diese Hemmung selbst dann fand, wenn er die Herzwirkung des Vagus durch Atropin ausschaltete.

c) Die Wirkung der Vagusreizung auf Magenperistaltik und Magenentleerung.

Ein Effekt der Reizung des Vagus auf die Motilität des Magens war fast in allen Versuchen deutlich nachweisbar. Nur bei vier von 24 dekapitierten Tieren fehlte er. Diese negativen Ergebnisse liessen sich jedoch leicht erklären.

In zwei von diesen vier Fällen konnte auch bei den stärksten Strömen keine Wirkung auf das Herz festgestellt werden. In dem dritten Fall handelte es sich um eine kranke Katze, die auf die Wismut-Kartoffelbreifütterung schon ohne Vagusreizung eine enorme Beschleunigung der Magendarmbewegungen aufwies. $\left({ }^{3 / 4}\right.$ Stunde nach Fütterung Kolonfüllung, $2^{3 / 4}$ Stunden nach Fütterung Defäkation von diarrhoischem, schleimigem Wismutstuhl.) In dem vierten Fall trat nach der Dekapitation eine sehr starke Blntung der Art. vertebrales auf.

In den 20 übrigen Versuchen zeigte das Magenbild während und nach der Vagusreizung ausnahmslos sehr starke Veränderungen hinsichtlich der Peristaltik und der Art der Entleerung. Diese Wirkung der Vagusreizung trat ebensowohl bei alleiniger Dekapitation mit intakten Splanchnici als nach beiderseitiger Splanchnicotomie ein. Prinzipielle Unterschiede liessen sich in unseren Versuchen in dieser Hinsicht nicht feststellen. Auch die für den Eintritt der Vaguswirkung nötige Reizstärke war nach Spanchnicotomie meist nicht geringer als bei Intaktheit dieser Nerven. Es können deshalb diese beiden Versuchsgruppen gemeinsam besprochen werden. Doch wird bei der Darstellung von Einzelheiten, die nicht immer beobachtet wurden, stets bemerkt, ob die splanchnischen Fasern durchschnitten waren oder nicht. In der Mehrzahl der Versuche waren sie erhalten.

Wie in den Normalversuchen an der Rückenmarkskatze geschildert, beginnt etwa $3 / 4$ Stunde nach der Fütterung der Pylorusteil leichte peristaltische Wellen zu zeigen und nach ungefähr $1 \frac{1}{2}$ Stunden etwas $W$ ismut in den Dündarm zu entleeren. Die 
übertretenden Güsse sind sehr gering und ganz vereinzelt. Nach den ersten paar Entleerungen sistiert gewöhnlich die Ergiessung auf eine mehr oder weniger lange Zeit, während die Peristaltik ruhig fortläuft. Reizt man die Vagi jetzt mit mittleren Strömen, also bis zur Pulsverlangsamung auf die Hälfte, so beobachtet man nach kurzer Latenz ganz aussergewöhnlich starke peristaltische Einschnürungen, nicht allein im Pylorusteil des Magens, sondern ebenso tief auch in dem sonst ziemlich bewegungslosen Fundus. Diese Kontraktionen laufen langsam dem Pylorus zu. Oft sind sie so stark, dass eine Verbindung zwischen den abgeschnürten Magensehatten nicht mehr sichtbar ist. Die Zahl dieser Einschnürungen, die gleichzeitig gesehen werden können, ist gering. Oft treten am ganzen Magen nur zwei bis drei mächtige peristaltische Wellen auf. In manchen Fällen teilt sich auch der Magen nur in zwei vollkommen voneinander getrennte Hälften, so dass er die Form eines S a nd u hrm a gens annimmt. Diese einzige tiefe Finschnürung bleibt jedoch nicht während der ganzen Dauer der Reizung unverändert bestehen. Sie läuft entweder dem Pylorus $\mathrm{zu}$, indem sie den Mageninhalt vor sich hertreibt, oder sie löst sich wieder und ist von zwei ebenso tiefen Kontraktionen, die den Magen in drei Teile scheiden, gefolgt. Es ist. besonders zu bemerken, dass diese einzige Kontraktion nicht immer in der Gegend des transversalen Bandes oder des sogenannten Sphincter antri pylori erscheint. Sie kann ebensogut
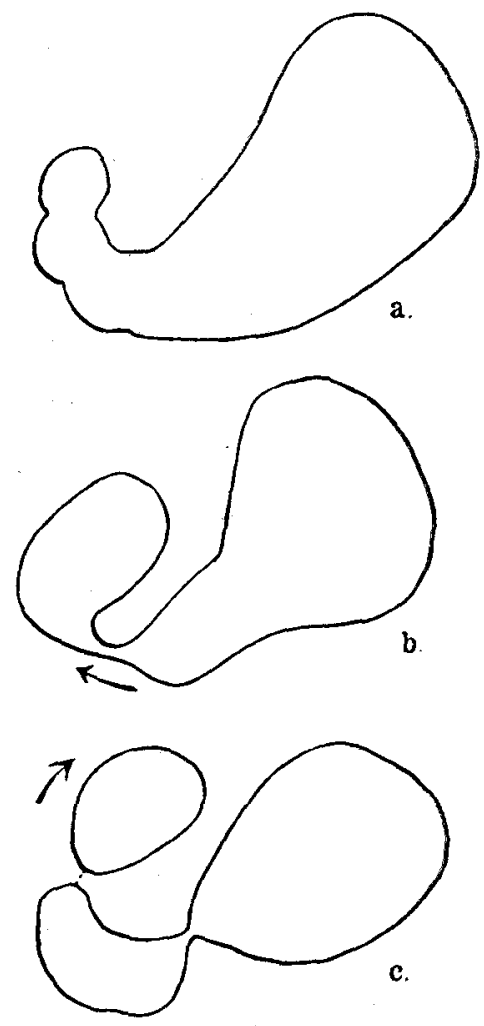

Fig. 3. (Auf $1 / 2$ verkleinert.)

12 h $05^{\prime}$. Dekapitation. $12^{\text {h }} 10^{\prime}$. Fütterung. $2 \mathrm{~b} 50^{\prime}$ bis $3 \mathrm{~h} 50^{\prime}$ leichte Peristaltik des Pylorusteils; im Dünndarm $3 \mathrm{~cm}$ Wismutschatten. $3^{\mathrm{h}} 50^{\prime}$. Vagusreizung, mit tle r e Stromstärke 11/2 Minuten lang. a) Magenbild vor der Reizung. b) Nach 15 Sekunden Reizung. Starke Kontraktion am Angulus, welche pyloruswärts wandert. c) Nach 20 Sekunden Reizung. VollkommeneAbschnürung eines Ballensim Pylorusteil und fast vollkommene Einschnürung in der Magenmitte. Beide Wellen befördern den abgeschnürten Mageninhalt durch den sich öffnenden Pylorus in das Duodenum. 
im Fundus auftreten, so dass zwei Drittel des Magens pyloruswärts abgeschnürt sind, oder sie kann am distalsten Ende des Pylorusteiles nur einen einzigen kleinen Ballen abschnüren.

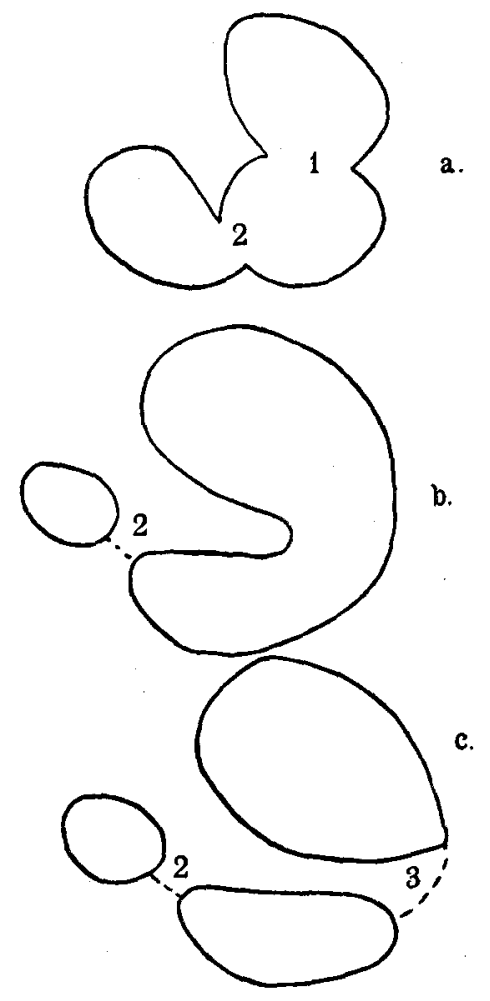

Als Beispiel für die mannigfaltigen Bilder, die hierbei entstehen können, dienen die umsteheud abgebildeten Schirmpausen (Fig. 3).

Derselbe Versuch. (Fig. 4.)

4 h. Vagusreizung mit $\mathrm{s} \mathrm{ch} \mathrm{w} \mathrm{a} \mathrm{ch} \mathrm{e} \mathrm{r}$ Stromstärke etwa $1 / 2$ Minuten lang. (Keine Pulsverlangsamung.) a) Starke Einschnürungen bei 1 u. 2. b) Die Einschnürung 1 löst sich, 2 wandert nach dem Pylorus, einen Ballen ganz abschnürend. c) 2 unverändert, an der Stelle von 1 eine dritte erneute vollkommene Einschnürung. Dreiteilung des Magens. d) Die Kontraktion 2 löst sich. Zweiteilung des Magens. Keine Öffnung des Pylorus.

Fig. 4. (Auf $1 / 2$ verkleinert.)

Die tiefen peristaltischen Kontraktionen lassen sich jedoch nicht bei gleichbleibender kontinuierlicher Reizung beliebig lange hervorrufen. Meist hört, wenigstens bei mittleren Strömen, nach etwa $1^{1 / 2}-2$ Minuten der Vaguseffekt auf, und der Magen reagiert kaum noch auf die Reizung. Wenn man aber den Strom einige Zeit, Sekunden bis Minuten, unterbricht und dann wieder reizt, kann man die Einschnürungen oft lange Zeit hindurch in ausgeprägtester Weise hervorrufen. Diese unterbrochene Reizung gab häufig die besten und regelmässigsten Resultate. Ausserdem war es unverkennbar, dass nach wiederholten Reizungen oft schou Reizstärken wirksam wurden, die vorher noch keinen Einfluss auf den Magen gehabt hatten. So kam es vor, dass für den ersten Reizeffekt Stromstärken 
notwendig wurden, die den Puls stark verlangsamten, dass man aber später, um denselben Effekt zu bekommen, auf Stromstärken heruntergehen konnte, die keine deutliche Pulsverlangsamung erzeugten. Auch in dem Versuch, dem die Fig. 3 und 4 entnommen sind, war dies der Fall. Dass man oft mehrere Male vergeblich den Vagus reizen muss, um eine deutliche Wirkung zu erhalten, stellten auch Bayliss und Starling am Dünndarni des Hundes fest.

Die tiefen, sich auf den ganzen Magen erstreckenden Kontraktionen waren bei mittleren Strömen die gewöhnliche Wirkung. Sie fanden sich bei erhaltenen Nn. splanchnici ebenso wie nach Splanchnicotomie. Allerdings ist beim Auftreten von nur einer tiefen Einschnürung die Zweiteilung des Magens (Sanduhrform) häufiger in den Fällen, in denen die Splanchnici erhalten sind. Ohne Vagusreizung waren sie in den Normalversuchen nie, auch nicht annähernd zu finden. Verwendet man zur ersten Reizung jedoch nur schwache Ströme, so kann in vielen Fällen nur eine Verstärkung der gewöhnlichen Peristaltik erreicht werden. Die so verstärkten peristaltischen Wellen gehen dann gewöhnlich nicht weiter auf den Fundus über. Sie führen auch nicht zu völliger Abschnürung des Magens in zwei oder drei Teile. Nur in der Nähe des Pylorus schnüren sie den Wismutinhalt in runden Ballen voneinander ab. Besonders schön war diese leichte Verstärkung und Vertiefung der normalen Peristaltik in einigen Fällen von Dekapitation und gleichzeitiger Splanchnicotomie zu verzeichnen. Notwendig für das Auftreten dieser verstärkten Peristaltik infolge von Vagusreizung war aber die Splanchnicotomie nicht. (Fig. 5.)

Die Verlaufsrichtung der oben geschilderten einzelnen tiefen Kontraktionen nach mittleren Strömen sowie der zahlreichen verstärkten peristaltischen Wellen nach schwachen Strömen war stets pyloruswärts. Nicht ein einziges Mal wurden Wellen beobachtet, die vom Pylorus zur Cardia hingingen.

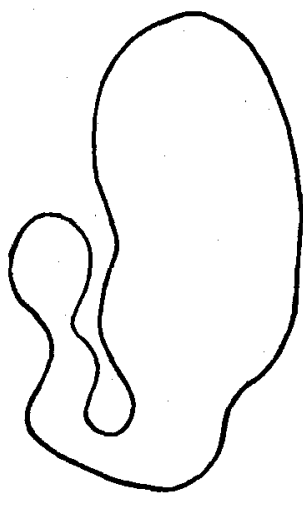

a.

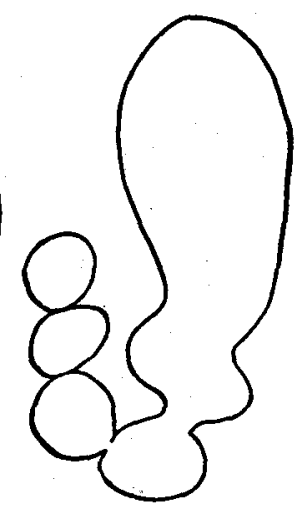

b.
Fig. 5. Dekapitation und Splanchnicotomie. (Auf $1 / 2$ verkleinert.)

a) 5 h $45^{\prime}$. Normale Peristaltik. b) 5 h $50^{\prime}$. Verstärkte Peristaltik infolge von Vagusreizung. (Schwache Ströme; kaum merkliche Pulsverlangsamung.) 
Auch bei einem Tier, bei dem sich bei der Sektion eine sehr starke Verziehung und Stenosierung des Pylorus infolge eines Tumors fand, war nach Vagusreizung keine antiperistaltische Bewegung sichtbar. Der Magen lag in diesem Falle noch 4 Stunden nach der Fütterung völlig bewegungslos. Nach $4^{1 / 2}$ Stunden wurde der Vagus gereizt. Nach mehreren wiederholten Reizungen mit mittleren bis starken Strömen traten tiefe Kontraltionen auf, die zu einer massenhaften Entleerung des Mageninhaltes in den Dünndarm führten. Fig. 6 gibt die eigentümlichen Kontraktionen nach Vagusreizung in diesem Falle wieder.

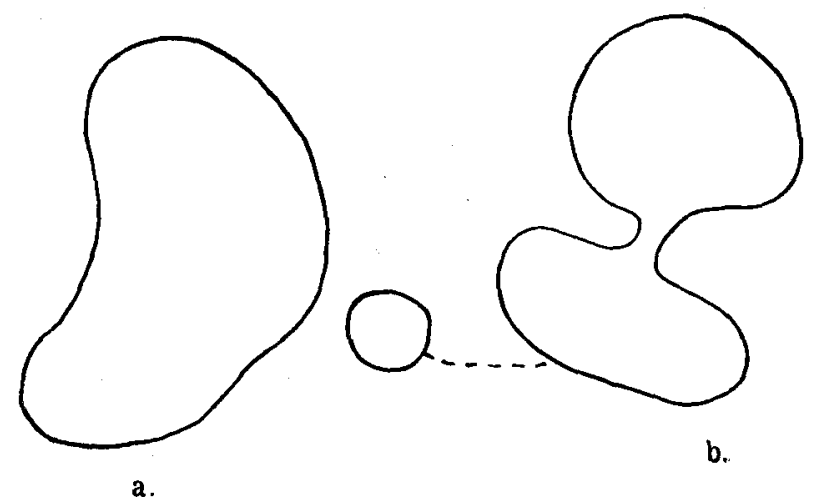

Fig. 6. $a$ vor Vagusreizung, $b$ während Vagusreizung. (Auf $1 / 2$ verkleinert.)

Ein von der gewöhnlichen Wirkung vollständig abweichendes Verhalten des Magens auf Vagusreizung konnte in zwei Fällen konstatiert werden. Hier waren die schwachen und mittleren Reizstärken unwirksam. Reizte man jedoch bis zum Herzstillstand, so nahm der vorher langgestreckte Magenschatten eine kreisrunde Form an. Man hatte den Eindruck, als ob der ganze Pylorusteil sich tonisch kontrahiert habe und der ganze Mageninhalt in den kuglich geformten Fundusteil gepresst sei. Setzte man dann mit diesem starken Strome aus, stellte sich allmählich das alte normale Magenbild wieder her.

Die Zeitdauer zwischen dem Einschalten des elektrischen Stromes und der Wirkung auf den Magen schwankte gewöhnlich zwischen 10 und 40 Sekunden. War nach 60 Sekunden kein Effekt eingetreten, fehlte er meist ganz. Nach wiederholten Reizungen, besonders kurz hintereinander, wurde diese Latenzzeit erheblich kürzer. Es erschienen dann oft schon unmittelbar nach der Reizung Bewegungsänderungen.

Nach den Untersuchungen von $\mathrm{Page} \mathrm{May}$ ist normalerweise die erste Wirkung der Vagusreizung Hemmung der rhyth- 
mischen Kontraktionen und Naçhlassen des Tonus; bei meinen Versuchen war nach schwachen Strömen diese Hemmung nicht deutlich sichtbar. In einigen Fällen schien die normale Peristaltik ohne weiteres in die verstärkte überzugehen. In diesen Fällen war mit
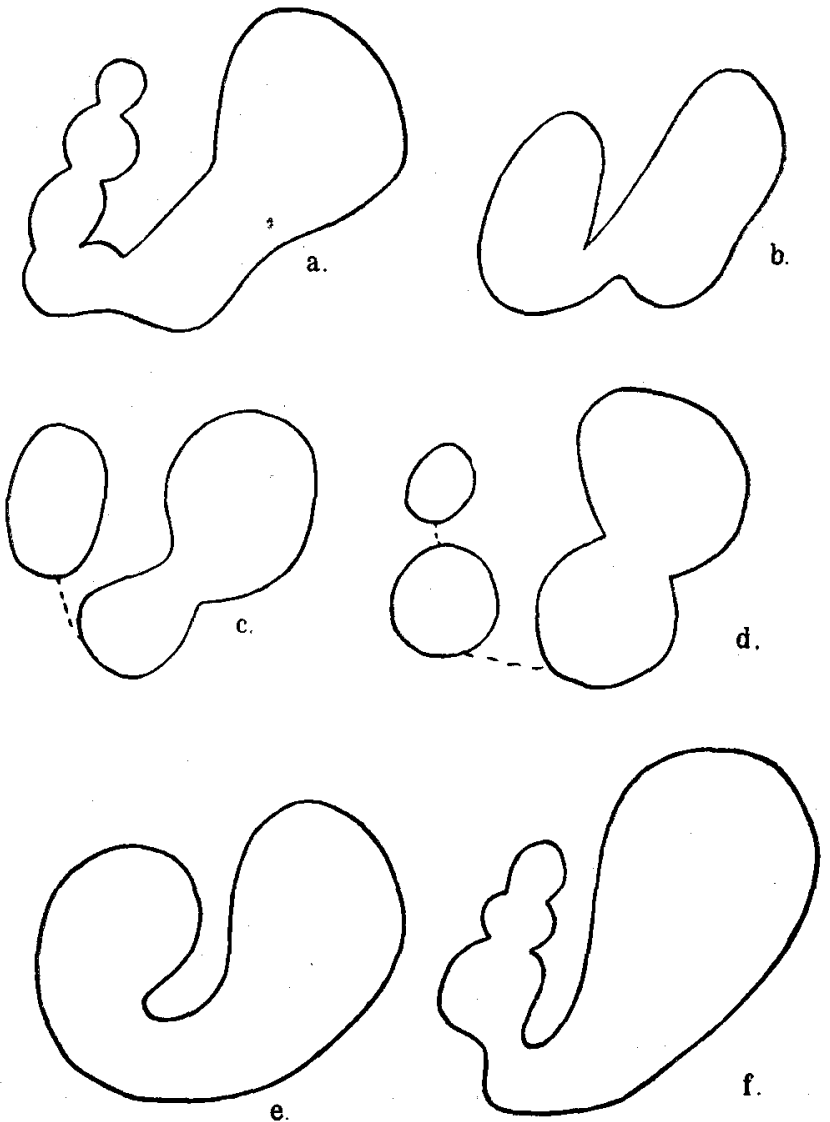

Fig. 7. a) 6 h $12^{\prime}$. Normale lebhafte Pylorusteilperistaltik. b) 6h $13^{\prime}$. 10 Sekunden nach Einsetzen der Vagusreizung mit mittleren Strömen. Sistierung der einzelnen peristaltischen Wellen. Der Magen hat sich aber schon kontrahiert. Einige Sekunden vorher bot er ein Bild wie bei e. c) 15 Sekunden nach Einsetzen der Vagusreizung. d) 25 Sekunden nach Einsetzen der Vagusreizung. e) 30 Sekunden nach A ufhören der Vagusreizung. Vollständige Bewegungslosigkeit des Magens. f) $1 / 4$ Stunde nach Aufhören der Vagusreizung. Wieder normale Peristaltik. (A uf $1 / 2$ verkleinert.)

unserer Versuchstechnik kein Sistieren der rhythmischen Kontraktion festzustellen. In anderen Fällen war die normale Peristaltik schon so schwach, dass eine Entscheidung über diese Frage nicht getroffen werden konnte. Bei mittleren Strömen war jedoch diese anfängliche Hemmungswirkung auf die peristaltischen Wellen fast immer nach- 
zuweisen, besonders nach Splanchnicotomie, da hierbei die normale Peristaltik sehr lebhaft und deutlich zu sein pflegte. (Fig. 7.)

In allen Versuchen, in denen diese anfängliche Erschlaffung der normalen Magenmotilität $\mathrm{zu}$ beobachten war, war sie jedoch stets nur vorübergehend. Sie dauerte wenige Sekunden, und dann traten sehr heftige Erregungen der Peristaltik auf. Wie aus Fig. $7 e$ hervorgeht, war der nächste Effekt der Ausschaltung des Reizes wieder eine Erschlaffung des Magens, der dann einige Zeit später seine normale Peristaltik wieder gewann (s. Fig. $7 f$ ). Das ist jedoch nicht immer der Fall. Manchmal ging die abnorm starke Peristaltik noch eine oder mehrere Minuten weiter und dann allmählich in normale Peristaltik über. In anderen Versuchen blieb die Erschlaffung des Magens nach mehrmaligem Vagusreiz bestehen. Es stellten sich dann spontan überhaupt keine oder nur ganz geringe Bewegungen wieder her, falls man nicht von neuem reizte. Es fiel auf, dass diese Schädigung der spontanen Motilität als Folge wiederholter Vagusreizungen fast nur bei intakten Splanchnici auftrat. Überall da, wo die Splanchnici durchschnitten waren, erreichte auch in den Pausen zwischen den einzelnen Reizungen die Peristaltik schnell wieder ihre normale Grösse, solange sich noch Inhalt in dem Pylorusteil befand.

$\mathrm{Ob}$ auch durch die Vagusreizung eine Veränderung des gesamten Magent on us hervorgerufen wird, kaun mit dem Röntgenverfahren nicht eindeutig bestimmt werden. Doch scheinen die auffallenden Veränderungen der Form des Magenschattens für eine solche Annahme zu sprechen. Meist trat nach Vagusreizung, bevor noch starke Peristaltik erschien, eine Verkleinerung des gesamten Magenschattens ein. Nach Aufhören der Reizung zeigte der Schatten dann wieder die alte Gestalt. Es kann sein, dass diese Verkleinerung auf einer Rundung des ganzen Magens, einer Zunahme des dorsoventralen Durchmessers beruhte. Vielleicht wurde der Magen auch dem Schirm näher gerückt, und dadurch erschien der Schatten und das Magenvolumen kleiner (s. Fig. 7). Jedenfalls gibt das Röntgenbild darüber keinen sicheren Aufschluss.

Wir schliessen aus unseren Versuchen, dass die elektrische Reizung des. Vagus hochgradige Verstärkung der Magenbewegungen hervorruft. Diese Bewegungsänderung besteht bei schwacher Stromstärke in einer vertieften und beschleunigten Peristaltik. Nach mittleren Strömen treten sehr heftige peristaltische Einschnürungen auf, die sich auf 
den ganzen Magen erstrecken und ihn in mehrere getrennte $A b$ schnitte $\mathrm{zu}$ teilen vermögen. Alle Einschnürungen laufen peristaltisch dem Pylorus zu. Diesen verstärkten Bewegungen kann eine kurze Hemmung vorausgehen.

Welchen Effekt hat nun diese Erregung der Peristaltik auf die Magenentleerung?

Wir haben gesehen, dass die starken Kontraktionen grosse Mengen von Wismut nach dem Pylorus hinbeförderten. Verfolgte man eine solche Welle, so konnte man gewöhnlich beobachten, wie kurz vor ihrer Ankunft am Pylorus sich plötzlich dessen Sphinkter öffuete und nun die ganze abgeschnürte Wismutmenge in einem mächtigen Ergusse in das Duodenum übertrat. Nachdem dies geschehen war, schloss sich der Pylorus wieder, um bei dem Herannahen der nächsten Welle sich wieder zu öffnen und nach Durchlassung ihres Inhaltes zu schliessen. Diese Öffnung des Pylorus konnte zeitweise relativ lange dauern, je nachdem eine grosse oder kleine Menge Mageninhalt durch die starken Kontraktionen abgeschnürt war. So kam es vor, dass in manchen Fällen, in denen durch eine grosse Einschnürung, etwa in der Magenmitte, der Mageninhalt in zwei Abschnitte geteilt war, der Pylorus so lange offen stand, bis der ganze Inhalt der abgeschnürten Seite in das Duodenum hinüber gepresst war. Setzte man nun plötzlich während dieses Übertritts von Wismutbrei mit der Reizung aus, so erfolgte auch jetzt erst dann die Schliessung des Pylorus, wenn die ganze Welle ihren Inhalt entleert hatte. Diese Öffnung und Schliessung des Sphincter pylori konnte auch nach Ausschaltung des Vagusreizes beobachtet werden, wenn die starke Peristaltik noch einige Minuten anhielt. Niemals öffnete sich der Pylorus auf Vagusreiz schon sofort, wenn am Magen die ersten tiefen Kontraktionen erschienen, sondern immer erst dann, wenn eine solche Kontraktion ihm eine mehr oder weniger grosse Menge von Mageninhalt zugeschoben hatte. Man gewann so den Eindruck, dass der Sphincter pylori in seiner Rhythmik nicht unmittelbar abhängig war von der Reizung des Vagus, sondern erst sekundär durch die starke Peristaltik eröffnet wurde. Wie weit jedoch hier chemische Reflexe mitspielten, speziell Säurereflexe, konnte mit unserem Verfahren nicht beurteilt werden.

Ähnliche Verhältnisse konnte ich gelegentlich auch am Sphincter cardiae sehen. Wenn nach der Fütterung noch Wismutbrei in dem Ösophagus zurück- 
geblieben war, so konnte man in seinem untersten Drittel leichte peristaltische Wellen bemerken, die aber nicht imstande waren, den Inhalt durch die Kardia zn befördern. Reizte man den Vagus mit mittleren Strömen, so brachten eine oder melirere starke peristaltische Wellen den ganzen Wismutbrei durch die sich öffnende Kardia in den Magen. Bei leerem Ösophagus blieb die Kardia in allen Fällen der Vagusreizung fest geschlossen. Ein Zurücktreten des Wismutbreies in den Osophagus als Folge der Vagusreizung konnte nie beobachtet werden.

Die Beförderung des Mageninhaltes in den Dünndarm durch Vagusreizung hatte jedoch ihre Grenzen. Wenn grosse Mengen von Wismutbrei in das Duodenum ubergetreten waren, so gelang es plötzlich nicht mehr, eine Öffnung des Pylorus zu erreichen. Obwohl noch sehr tiefe Peristaltik erzeugt werden konnte, war der Wiederstand des Pylorus nicht mehr zu überwinden. Man sah, wie die Wellen bis vor den Pylorus hinliefen und dann wieder erschlafften.

Ein Beispiel für dieses Verhalten bieten die Fig. 3 und 4. Bei Fig. 3 ergossen sich die abgeschnürten Ballen jedesmal in das Duodenum; 10 Minuten später (Fig. 4) blieb bei gleichstarker Peristaltik der Sphinkter geschlossen.

Doch gelang es dann nach einiger Zeit wieder durch Reizung des Vagus Übertritt von Mageninhalt in den Dünndarm zu erzielen. Wahrscheinlich ist auch dieser vorübergehende feste Verschluss des Pylorus auf den bekannten Reflex im Duodenum zu beziehen.

Die eben dargestellten Beobachtungen entsprachen dem Verhalten der Magenentleerung auf mittlere Ströme. Bei schwachen Strömen waren die Ergebnisse nicht so gleichmässig. In einer Reihe von Versuchen fand man häufig, wie die einzelnen verstärkten Wellen in beschleunigtem Rhythmus Wismutgüsse in das Duodenum be-

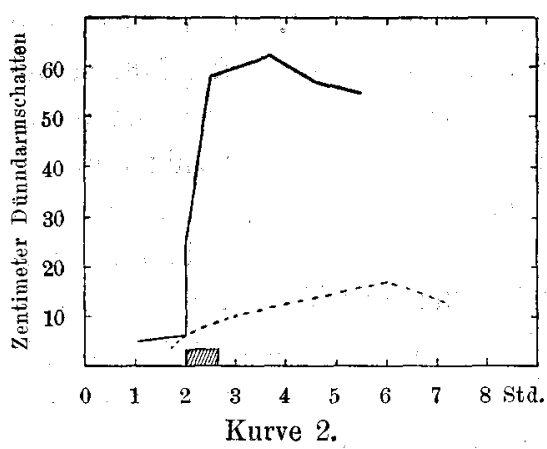

- Dekapitation mit Vagusreizung.

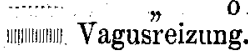

förderten; in anderen Experimenten war wohl eine verstärkte Magenperistaltik, aber kein Übertritt in den Darm nachzuweisen. Schaltete man dann mittlere Ströme ein, so war dieser Effekt jedesmal zu erreichen. Der Übertritt von Wismutbrei in den Dünndarm bei schwachen Strömen war häufiger nach Splanchnicotomie als bei erhaltenen Splanchnici 
Der Einfluss der Vagusreizung auf den Ablauf der Verdauungsbewegungen. 585

majores $z u$ beobachten. Jedenfalls war es immer möglich, mit mittleren Strömen den Magen bis zu einem erheblichen Grade zu entleeren und den Dünndarm stark zu füllen.

Kurve 2 gibt einen Versuch wieder, indem etwa 1 Stunde nach der Fütterung etwas Wismut in den Dünndarm übergetreten war. Nach 2 Stunden hatte sich dieser Füllungszustand des Darms nicht wesentlich vermehrt. Dann wurde der Vagus wiederholt gereizt. Man sieht, wie innerhalb einer $1 / 2$ Stunde die Füllung des Dünndarms ausserordentlich zugenommen hat, mit einer Geschwindigkeit, wie man sie beim normalen Tier selten findet. Zum Vergleich ist punktiert die durchschnittliche Normalkurve der Dünndarmfüllung bei der Rückenmarkskatze ohne Vagusreizung beigefügt.

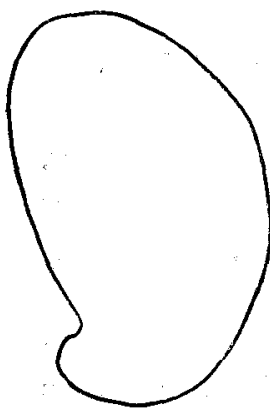

a.

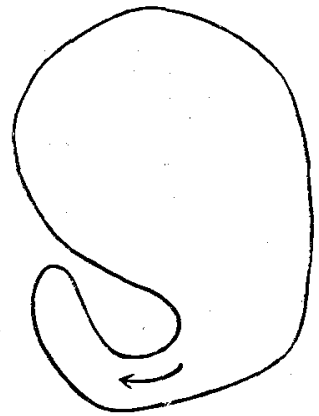

b.
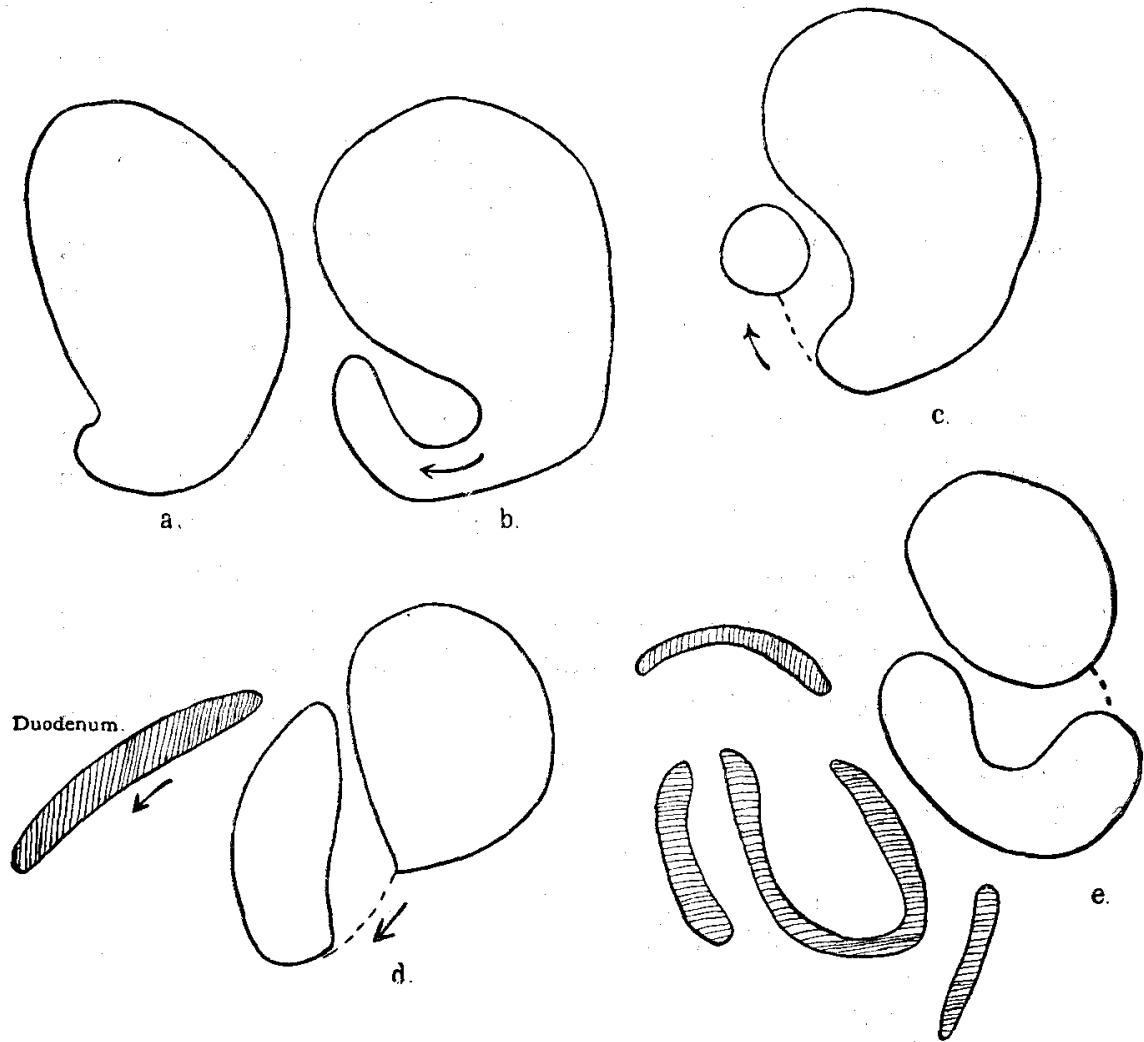

Fig. 8. (Auf $1 / 2$ verkleinert.) $11 \mathrm{~h} \cdot 15^{\prime}$. Dekapitation. $2 \mathrm{~h} 15^{\prime}$. Präparation und Abbindung der Vagi. Anlage der Elektroden. 2 h $52^{\prime}$ bis 53'. Einführung des Wismutbreies mittelst Sonde. $2 \mathrm{~h} 54^{\prime}$. Fundus gefüllt. Pylorusteil angedeutet. (Fig. 8 a.) - Vagusreizung (schwache Stromstärke) obne Effekt. $2^{\text {h }} 57^{\prime}$. Vagusreizung (mittlere Stromstärke) 1 Minute. Füllung des Pylorusteils, auf dem Schirm beobachtet. (Fig, 8 b.) -2 h $59^{\prime}$. Vagusreizung (mittlere Stromstärke) 1 Minute. Nach 30 Sekunden Abschnürung eines Wismutteils. (Fig. 8 c.) - Nach 50 Sekunden Übertritt dieses Ballens in den Dünndarm, Erneute peristaltisch fortwandernde Einschnürung in der Magenmitte. (Fig. 8 d.) $-3 \mathrm{~h}^{\prime} 0^{\prime}$. Einschnürung der Magenmitte. Weitere Füllung des Dünndarms $(19 \mathrm{~cm})$. (Fig. 8.e.) 
Der vorstehend geschilderte Versuch beweist die Wirkung des Vagus auf die Magenentleerung einige Zeit nach der Fütterung, wenn die normale Peristaltik und Dünndarmfüllung schon begonnen hat. Diese Wirkung ist jedoch auch vorhanden, wenn unmittelbar nach der Einfuhrung des Wismutbreies der Vagus gereizt wird. Der Vorgang der Magenentfaltung und ersten Entleerung in das Duodenum, der bei der dekapitierten Katze gewöhnlich 1-2 Stunden dauert, kann dann schon in wenigen Minuten geschehen. Die hier abgebildeten Schirmpausen wurden bei einem Versuch begonnen, in dem die Vagusreizung sofort nach der Sondenfütterung begann. Die Splanchnici waren nicht durchschnitten. (Fig. 8.)

Es gelingt also, den Magen durch unmittelbar nach der Fütterung erfolgende Vagusreizung in $6-7$ Minuten zur ersten Entleerung $\mathrm{zu}$ bringen. Dieser Vaguseinfluss erscheint besonders auffällig, wenn man damit den Verlauf der Magenentfaltung und -entleerung vergleicht, wie er in dem Normalversuch auf S. 563 (Abb. 1) dargestellt ist.

Es wurde oben betont, dass eine vollständige Entleerung des Magens an der dekapitierten Katze niemals stattfand, auch nicht, wenn die Splanchnici durchschnitten waren. Nach Vagusreizung gelang dies in zwei von 24 Fällen. In diesen beiden Fällen waren die Splanchnici erhalten. In den übrigen Versuchen enthielt der Fundus immer noch reichlich Wismutbrei. Dass aber ein Erfolg der Reizung noch nachweisbar war, wenn sich auch schon der Magen weitgehend entleert hatte, mit anderen Worten, dass die erregende Wirkung des Vagus nicht an einem bestimmten stärkeren Füllungszustand des Magens gebunden war, ging aus vielen Versuchen hervor (vgl. Fig. 9).

$11^{\text {h }} 10^{\prime}$. Dekapitation. Splanchnicotomie.

$1^{\text {h }} 50^{\prime}$. Fütterung.

a) 11 Stunden nach der Fütterung Fundus noch etwas gefïllt. Pylorusteil fast leer. Übertritt von Wismut in den Dünndarm sistiert seit längerer Zeit.

b) 40 Minuten nach Vagusreizung; mehrfache Abschnürungen und Übertritt von Wismutbrei in grossen Schüben.

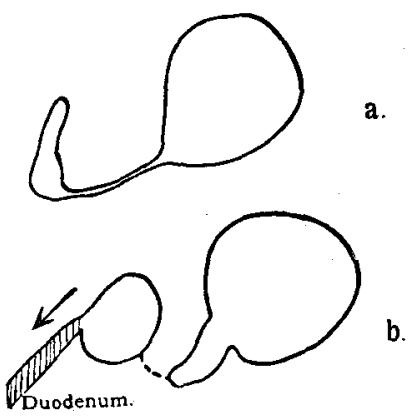

Fig. 9. (Auf $\mathbf{1}_{1 / 2}$ verkleinert.)

Der Effekt der Vagusreizung auf die Magenentleerung lässt sich folgendermassen zusammenfassen. Reizung mit mittleren Strömen 
befördert den Mageninhalt in mächtigen Schüben in den Dünndarm. Bei Reizung mit schwachen Strömen ist dieser Erfolg nicht immer vorhanden. Ist infolge wiederholter, kurz aufeinanderfolgender Reizungen eine grössere Menge Mageninhalt in das Duodenum übergetreten, so schliesst sich der Pylorus für einige Zeit und lässt trotz fortdauernder Vagusreizung nichts mehr passieren. Die erste Entleerung des Magens in den Darm, die normalerweise an der Rückenmarkskatze 1--2 Stunden nach der Fütterung auftritt, kann durch Vagusreizung in 6-7 Minuten $\mathrm{n}$ ach $\mathrm{F} u ̈ t$ terung erreicht werden. Eine völlige Entleerung des Magens ist selten. Zuracktreten von Mageninhalt in den Ösophagus findet bei Vagusreizung nie statt. Die Cardia bleibt fest geschlossen, vorausgesetzt, dass der Ösophagus leer ist. Befindet sich im Ösophagus Inhalt, so wird er bei Vagusreizung durch starke peristaltische Kontraktionen durch die sich öffnende Cardia in den Magen befördert.

d) Die Wirkung der Vagusreizung auf die Dünndarmbewegungen und die Weiterbeförderungdes Dünndarminhaltes.

In allen Versuchen, in denen eine Beeinflussung der Magenbewegungen durch die Vagusreizung festzustellen war, ergab sich auch eine deutliche Wirkung auf den Dünndarm. Sie bestand auch hier in einer über die Norm hinausgehenden Beschleunigung der Bewegungen. Diese Darmwirkung des Vagus beruhte nicht auf einer Fortplanzung der Kontraktionen vom Magen her. Bei vollkommen bewegungslosem Magen, ohne dass Magenperistaltik oder Übertritt von Wismutinhalt in das Duodeum stattfand, konnten sehr heftige Darmbewegungen das einzige Resultat der Vagusreizung sein. In anderen Fällen trat heftige Peristaltik des Dünndarms nach Reizung des Vagus auf, lange bevor die Peristaltik des Magens einsetzte. Die Darmwirkung konnte schon 20 Sekunden nach Einsetzen der Reizung sehr deutlich sein. während in demselben Einzelversuch die Magenwirkung erst nach 60 Sekunden eintrat. $\mathrm{Zu}$ ähnlichen Resultaten kamen auch Bayliss und Starling, indem sie durch eine Ligatur hinter dem Pylorus oder vor dem Beginn des Jejunums eine Fortpflanzung der Bewegungen der höher gelegenen Abschnitte des Verdauungsrohres verhinderten. Auch die Reizstärke, die Darmbewegungen hervorrief, war nicht immer dieselbe, wie die, welche zu einer deutlichen Wirkung auf den Magen nötig war. Manchmal war es leichter, 
einen Effekt auf den Magen hervorzurufen, manchmal trat die Darmwirkung leichter ein. Die Verbältnisse variierten in dieser Hinsicht bei den einzelnen Tieren sehr.

Die erregende Wirkung des Vagus auf die Dündarmbewegungen zeigte sich vor allem in einer $Z$ unahme der Peristaltik. Seltener war eine Beschleunigung der rhythmischen Segmentationen. Die Fortbewegung der peristaltischen Welle konnte mit ausserordentlich grosser Schnelligkeit vor sich gehen. In einzelnen Fällen betrug die Gesehwindigkeit der einzelnen Welle $4 \mathrm{~cm}$ und mehr in 1. Sekunde. Eine einzige peristaltische Einschnürung konnte oft eine enorme Wismutmenge vor sich hertreiben. Die Länge der so vorwärts geschobenen Wismutsäule wechselte, doch war sie meist grösser als normal. In einem Falle wanderte ein über $13 \mathrm{~cm}$ messendes Wismutstück darmabwärts. Das Bild der so nach Vagusreizung durch die verschiedenen Darmsehlingen schiessenden Wismutmassen erinnerte dann lebhaft an die Bewegungen einer Schlange. Niemals sah man jedoch, dass eine einzige peristaltische Welle kontinuierlich durch den ganzen Dünndarm bis zum Colon lief. Die beschleunigten peristaltischen Wellen waren auch nach Splanchnicotomie $z \mathfrak{u}$ beobachten.

Van Braam-Houkgeest ${ }^{1}$ ) erhielt ähnliche Bewegungen am Darm von Kaninchen im Kochsalzbad bei Vagusreizung nach vorhergegangener Durchschneidung der Splanchnici und bezeichnete sie als Rollbewegungen. Meltzer und $A$ ue $\mathrm{r}^{2}$ ) fanden, dass bei gleichzeitiger Applikation von Calciumchlorid und Ergotin Rollbewegungen vom Pylorus ohne Aufhören bis zum Coecum liefen (complete peristaltic rush) und dass Durchschneiden der Vagi das Auftreten dieser kompletten Rollbewegungen verhinderte.

Die geschilderten ausgedehnten peristaltischen Wellen waren jedoch nicht immer mit Sicherheit zu erzeugen. Oft trat auf Vagusreizung eine Peristaltik ein, die sich nur durch eine leichte Beschleunigung von einer normalen peristaltischen Darmwelle unterschied.

Was die rhythmischen Segmentationen anbetrifft, so konnte in einzelnen Fällen ein direkt errregender Einfluss des Vagus nachgewiesen werden. Meist war es dann so, dass die Peristaltik ausblieb, wohl aber eine beschleunigte und vermehrte Teilung des Wismutinhaltes in einzelne Segmente eintrat. Besonders bei einem Versuche mit Splanchnicotomie war diese Erscheinung durch Vagus-

1) van Braam-Houkgeest, l: c.

2) Meltzer and Auer, l.c. 
reizung mit Regelmässigkeit hervorzurufen. Häufiger kam es vor, dass durch die Reizung des Vagus ein Wismutabschnitt schnell weiter wanderte, nach Aussetzen des Reizes liegen blieb und sich nun erst lebhaft segmentierte. In anderen Fällen endlich konnten beide Bewegungsarten an einem und demselben Darmabschnitt hervorgerufen werden. Durch eine starke peristaltische Welle wurde ein Wismutstück schnell fortgeschoben, und gleichzeitig während des Vorruckeus erschienen rhythmische Segmentationen.

Die Veränderungen der Dünndarmbewegungen durch Vagusreizung waren nicht in allen Darmabschnitten gleichmässig stark. In den oberen Dünndarmpartien, besonders im Duodenum, war die Vaguswirkung am deutlichsten. Im Ileum war nicht immer ein sichtbarer Effekt vorhanden. Die Füllung des Duodenums durch den Magen konnte verschieden sein. Entweder traten infolge der verstärkten peristaltischen Antrumwellen bei schwachen Strömen zahlreiche kleine Wismutballen über, die dann meist sehr schnell aus dem Duodenum verschwanden, oder es füllte sich durch die mächtigen Magenkontraktionen bei mittleren Strömen das Duodenum und die zunächst liegenden Abschnitte des Jejunums stark an. Setzte man dann mit dem Vagusreiz aus, so traten häufig sofort lebhafte rhythmische Segmentationen ein (Fig. 10).

In anderen Versuchen blieb der stark gefüllte Darm noch eine kürzere oder längere Zeit nach Ausschaltung des Reizies bewegungslos liegen. Erst allmählich traten wieder Fig. 10. (Auf $1 / 2$ verkleinert.) a) Während rhythmische und peristaltische Bewegungen auf. In ähnlicher Weise zeigte sich ja auch im

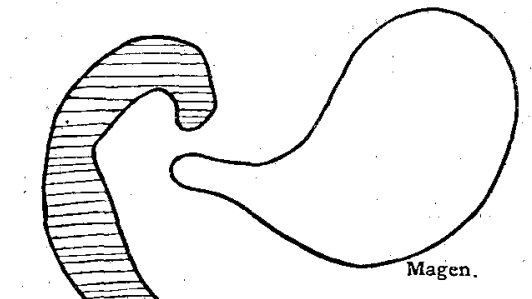

a.
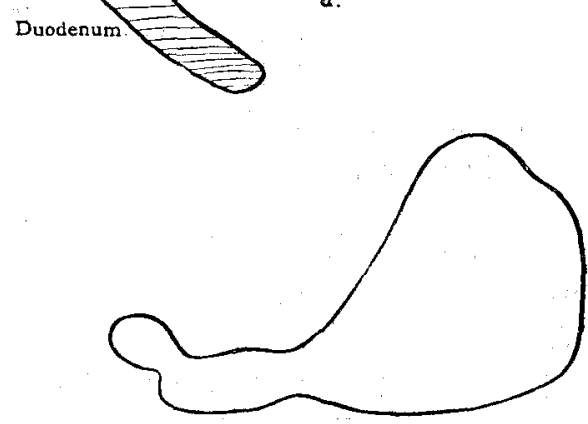

b.

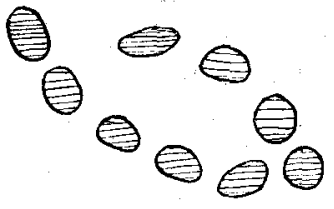

der Reizung des Vagus. Breiter bandförmiger Wismutschatten im Duodenum infolge starker Magenentleerung. b) 1 Minute nach Vagusreizung, sofortiges Eintreten von rhythmischen Segmentierungen. 
Magen öfters nach Sistieren der Vagusreizung mehr oder weniger ausgeprägte Erschlaffung.

Die Zeit, die der erste aus dem Magen ausgetretene Wismut, ballen brauchte, um das Colon zu erreichen, war nach Vagusreizungen sehr verschieden. Die schnellste Colonfüllung trat $1^{1 / 2}$ Stunde nach der Magenentleerung auf. Meist dauerte es länger, 3-4 Stunden, trotz wiederholter auf den Darm wirksamer Vagusreize. In anderen Fällen bestand wohl eine hocbgradige Dünndarmfüllung, ohne dass jedoch ein Übertritt in den Dickdarm erfolgte.

Kurve 3 zeigt die durch wiederholte Reizungen verursachte sehr starke Dünndarmfüllung. Im Colon ist nach 8 Stunden noch kein Wismut sichtbar.

In manchen Fällen konnte man während der Vagusreizung eine Zunahme der Colonfullung sehen. Es ist also der Übertritt durch die Ileocoecalklappe während der einzelnen Reizungen nicht behindert. In anderen Fällen gelang es nicht, durch Vagusreizung diesen Übertritt zu erzwingen. Iiese Verhältnisse waren bei intakten Splanchnicis und nach Splanchnicotomie gleich.

Zusammenfassend können

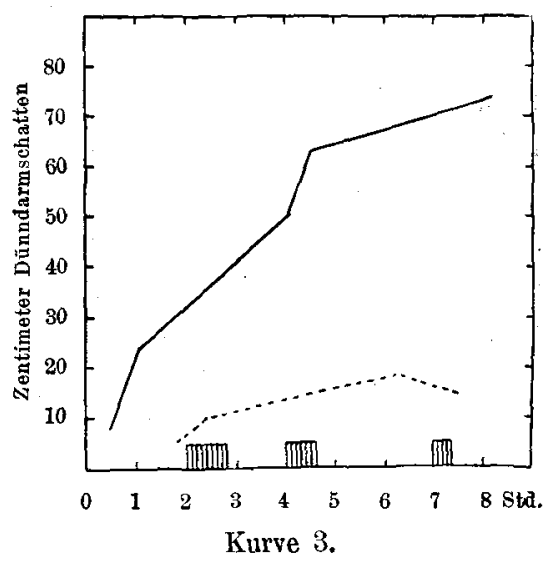

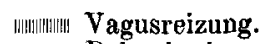

- Dekapitation mit Vagusreizung. wir also sagen, die Reizung des Vagus steigert die Bewegungen des Dündarms. Vor allem treten sehr ausgedehnte und beschleunigte peristaltische Wellen auf. Diese peristaltischen Wellen können eine grosse Strecke des Dünndarms durchlaufen und dabei erhebliche Mengen Wismutbrei vor sich herschieben. Ebenso kann sich die Wirkung des Vagus in einer Verstärkung der rhythmischen Segmentationen zeigen. Der Einfluss des Vagus auf die unteren Abschnitte des Dünndarms ist geringer als auf die oberen. So gelingt es nur in wenigen Fällen, den Transport des Ileuminhaltes in das Coecum zu beschleunigen. 
e) Versuche über das Verhalten des Dickdarms bei Reizung des Vagus.

Fast in allen Versuchen, in denen Dickdarmfüllung eingetreten war, blieben die Bewegungen dieses Darmabschnittes unbeeinflusst durch schwache oder starke Vagusreizung. Die Antiperistaltik des proximalen Colons konnte durch Vagusreizung weder aufgehoben noch hervorgerufen werden. Auch die gewöhnlich zwischen proximalem und distalem Colon befindliche Einschnürung änderte sich auf Vagusreizung nicht. Die Fortbewegung des Dickdarminhaltes erfuhr im allgemeinen keine Beschleunigung. Nur liess sich, wie oben erwähnt, zeitweise eine stärkere Füllung des proximalen Colons vom Ileum ber nach Vagusreizung feststellen. In zwei Fällen jedoch fand sich kurz nach Ausschalten der Reizung eine Kontraktion am proximalen Colon, die langsam distalwärts lief und eine Wismutmasse vor sich herschob. Da es möglich war, dass diese Kontraktion nur eine Folge von stärkerem Übertritt des Ileuminhaltes in das Coecum war, wurde in mehreren Versuchen der Vagus erst gereizt, nachdem sich die ganze Wismutmasse im Dickdarm befand.

Die Versuchsanordnung war dann folgende: Abends erhielt das für den Versuch bestimmte Tier die übliche Wismutmahlzeit. Am nächsten Morgen wurde durchleuchtet. Befand sich dann das Wismut vollständig im Colon, oder waren nur noch Dünndarmschatten von wenigen Zentimetern zu sehen, so wurde die Dekapitation vorgenommen. 1-2 Stunden nach der Dekapitation wurde bei gleichzeitiger Durchleuchtung der Vagus gereizt.

Es ergab sich, dass in keinem Falle während der oft $1 \frac{1}{2}$ Minuten und länger dauernden Vagusreizung irgendwelche Bewegungsänderungen eintraten. Die Antiperistaltik lief ruhig weiter. In einem von diesen drei Fällen sah man 6 Minuten nach der Reizung wieder stärkere Kontraktionen im proximalen Colon (Fig. 11). In den beiden anderen Fällen liess sich dann deutlich nachweisen, dass ähnliche Kontraktionen im proximalen Colon auftraten, wenn die letzten Reste des Wismutinhaltes aus dem Ileum in das Coecum übertraten. Einmal geschah dies, bevor überhaupt der Vagus gereizt war, in dem anderen Versuch setzte sich kurz nach der Vagusreizung die noch im Ileum liegende kleine Wismutsäule in Bewegung, trat in das Colon über, und im selben Moment bildeten sich die obigen Kontraktionen.

Da sich derartige Kontraktionen auch am lebenden normalen Tiere finden, sobald Ileuminhalt in das Colon übertritt, wie Cannon 
beschrieb, und sie nie während der Reizung, soudern immer erst einige Zeit später auftreten, sind sie wohl kaum direkt auf die Wirkung des Vagus zu beziehen.

Die Wirkungslosigkeit der Vagusreizung auf das Colon liess sich noch auf eine andere Weise deutlich demonstrieren. Nährklystiere können durch antiperistaltische Bewegungen des Colons durch die geöffnete Ileocoecalklappe bis in den Dünndarm befördert werden. $\mathrm{Cannon}$ verwandte zu seinen Röntgenuntersuchungen ein Klystier, bestehend aus $100 \mathrm{ccm}$ Milch, $2 \mathrm{~g}$ Stärke, $15 \mathrm{~g}$ Bismutum subnitricum und einem Ei. Je nachdem man das Ei mit kocht oder nicht, kann man einer mehr oder weniger konsistenten Brei erhalten. Bei Ein-

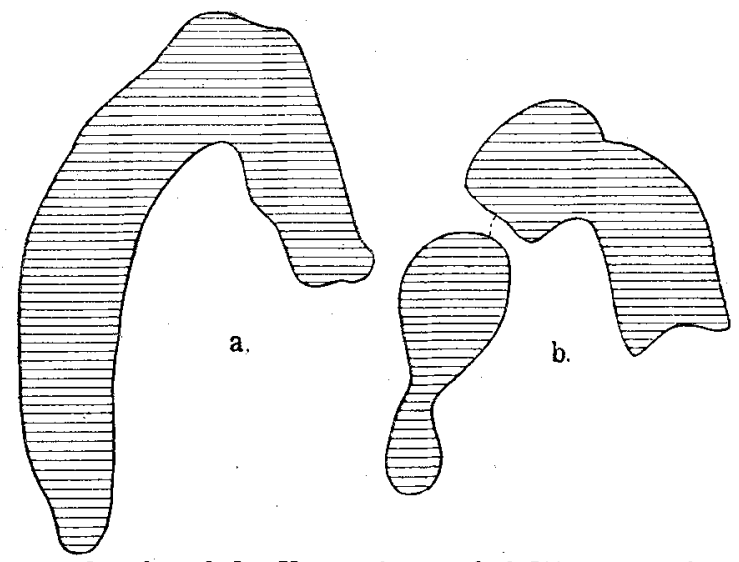

Fig. 11. $a$ vor und während der Vagusreizung. $b \quad 6$ Minuten nach Vagusreizung.

führung des Klysmas konnte er die Bewegungen des Colons und den Übertritt in den Dünndarm an der normalen lebenden Katze im Röntgenbild sehr deutlich verfolgen.

Versuche an der dekapitierten Katze ergaben, dass auch hier diese antiperistaltische Tätigkeit des Colons stattfand. Nach obigem Rezept bereitete Klystiere konnten an der Rückenmarkskatze bis zu $50 \mathrm{~cm}$ im Dünndarm aufwärts wandern (Sektion). Reizte man nun während der antiperistaltischen Füllung des Colons den Vagus, so ergab sich in keinem Falle eine sichtbare Änderung. Niemals traten abnorme Kontraktionen auf, niemals stockte das antiperistaltische Aufwärtswandern des Wismutgemenges.

\section{Zusammenfassung der Ergebnisse.}

I. Nach Abtrennung von Gehirn und Medulla oblongata (Dekapitation nach Sherrington) lässt sich an der künstlich geatmeten 
und erwärmten Rückenmarkskatze der gesamte Ablauf der Verdauungsbewegungen mit dem Röntgenverfahren verfolgen.

Die hierbei auftretenden Abweichungen vom normalen sind ausführlich geschildert. Sie stimmen im wesentlichen überein mit den Störungen, die Cannon an der lebenden vagotomierten Katze fand. Sie sind geringer, wenn beiderseits die oberen Nn. splanchnici durchschnitten werden.

II. Die Rückenmarkskatze eignet sich zur Untersuchung der Reizwirkung der efferenten Nerven auf die Verdaungsbewegungen besonders, da Einflüsse des Grosshirns oder der Narkose vermieden sind.

III. Die elektrische Reizung des peripheren Vagusstumpfes an der Rückenmarkskatze ruft abnorm starke Bewegungen des Magens und Dünndarms hervor. Diese Wirkung tritt auch ein bei Reizstärkeu, die noch keine Kreislaufstörung erzeugen. Die Wirkung. lässt sich ebensowohl bei erhaltenen Splanchnicis als nach Splanchnicotomie beobachten.

IV. Am Magen besteht die Wirkung der Vagusreizung bei schwachen Strömen in einer vertieften und beschleunigten Peristaltik. Bei mittlerer Stromstärke treten sehr heftige peristaltische Einschnürungen auf, die sich auf den ganzen Magen erstrecken und ihn in mehrere getrennte Abschnitte zu teilen vermögen. Alle Einschnürungen laufen peristaltisch dem Pylorus zu. Den verstärkten Bewegungen kann eine kurze Hemmung vorausgehen. Die starken peristaltischen Wellen befördern den Mageninhalt in sehr grossen Schüben in den Dünndarm. Der Pylorus öffnet sich bei dem Herannahen einer Welle und schliesst sich nach Entleerung ihres Inhaltes. Sind jedoch mehrere starke Entleerungen in das Duodenum erfolgt, so schliesst er sich kürzere oder längere Zeit und lässt trotz Weiterdauer der Vagusreizung und der Peristaltik nichts mehr passieren.

Die erste Entleerung des Magens in den Darm, die normalerweise an der Rückenmarkskatze 1-2 Stunden nach der Fütterung auftritt, kann durch Vagusreizung in 6-7 Minuten nach der Fütterung erreicht werden. Die Cardia bleibt fest geschlossen, vorausgesetzt, dass der Ösophagus leer ist. Ein Zurücktreten des Mageninhaltes in den Ösophagus findet bei Vagusreizung nicht statt.

V. Am Dünnd a rm treten nach Vagusreizung sehr ausgedehnte und beschleunigte peristaltische Wellen auf. Die Wellen können grosse Strecken des Dünndarms durchlaufen und dabei erhebliche 
594 Ph. Klee: Der Einfluss der Vagusreizung auf den Ablauf etc.

Mengen Darminhalt vor sich herschieben. Ebenso kann sich die Wirkung des Vagus in einer Beschleunigung der rythmischen Segmentationen zeigen. Der Einfluss des Vagus auf die unteren Abschnitte des Dünndarms ist geringer als auf die oberen. Es gelingt nur in wenigen Fällen, den Transport des Ileuminhaltes in das Coecum zu beschleunigen.

VI. Ein direkter Einfluss der Vagusreizung auf die Bewegungen des Dickdarms liess sich bei der Katze nicht nachweisen. 